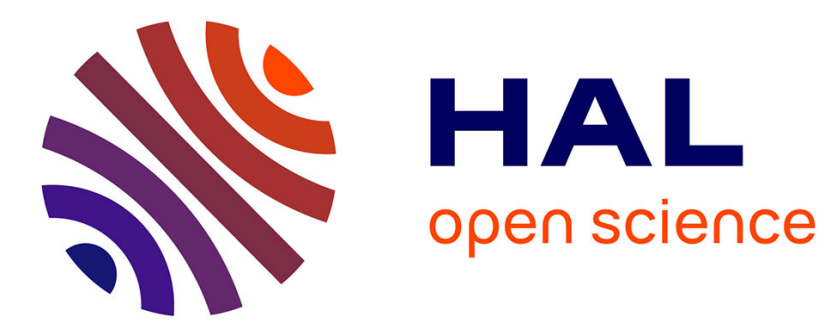

\title{
Persymmetric Adaptive Radar Detectors
}

Guilhem Pailloux, Philippe Forster, Jean-Philippe Ovarlez, Frédéric Pascal

\section{To cite this version:}

Guilhem Pailloux, Philippe Forster, Jean-Philippe Ovarlez, Frédéric Pascal. Persymmetric Adaptive Radar Detectors. IEEE Transactions on Aerospace and Electronic Systems, 2011, 47 (4), pp.23762390. 10.1109/TAES.2011.6034639 . hal-00659834

\section{HAL Id: hal-00659834 \\ https://hal-centralesupelec.archives-ouvertes.fr/hal-00659834}

Submitted on 4 Mar 2020

HAL is a multi-disciplinary open access archive for the deposit and dissemination of scientific research documents, whether they are published or not. The documents may come from teaching and research institutions in France or abroad, or from public or private research centers.
L'archive ouverte pluridisciplinaire HAL, est destinée au dépôt et à la diffusion de documents scientifiques de niveau recherche, publiés ou non, émanant des établissements d'enseignement et de recherche français ou étrangers, des laboratoires publics ou privés. 


\title{
Persymmetric Adaptive Radar Detectors
}

\author{
Guilhem Pailloux, Philippe Forster, Jean-Philippe Ovarlez and Frédéric Pascal
}

\begin{abstract}
In the general framework of radar detection, estimation of the Gaussian or non-Gaussian clutter covariance matrix is an important point. This matrix commonly exhibits a particular structure: for instance, this is the case for active systems using a symmetrically spaced linear array with constant pulse repetition interval. In this paper, we propose to use the particular persymmetric structure of the covariance matrix to improve the detection performance.

In this context, this paper provides two new adaptive detectors for Gaussian additive noise and nonGaussian additive noise which is modeled by the Spherically Invariant Random Vector. Their statistical properties are then derived and compared to simulations. The vast improvement in their detection performance is demonstrated by way of simulations or experimental ground clutter data. This allows for the analysis of the proposed detectors on both real Gaussian and non-Gaussian data.
\end{abstract}

\section{Index Terms}

Adaptive signal detection, Gaussian and non-Gaussian clutter, SIRV, covariance matrix estimation.

\section{INTRODUCTION}

The problem of adaptive radar detection requires the estimation of the clutter covariance matrix (CCM). In recent years, improvements of the associated estimation schemes have gained tremendous interest in

G. Pailloux is with the French Aerospace Lab (ONERA), DEMR/TSI, BP 72, 92322 Chatillon Cedex, France (email: guilhem.pailloux@onera.fr).

P. Forster is with the SATIE, ENS Cachan, CNRS, UniverSud, 61, av President Wilson, F-94230 Cachan, France (email: philippe.forster@satie.ens-cachan.fr).

J.P. Ovarlez is with the French Aerospace Lab (ONERA), DEMR/TSI, BP 72, 92322 Chatillon Cedex, France and with SONDRA, Supélec, Plateau du Moulon, 3 rue Joliot-Curie, F-91192 Gif-sur-Yvette Cedex, France (email: jeanphilippe.ovarlez@onera.fr).

F. Pascal is with SONDRA, Supélec, Plateau du Moulon, 3 rue Joliot-Curie, F-91192 Gif-sur-Yvette Cedex, France (email: frederic.pascal@supelec.fr). 
the radar community. For that purpose, the Sample Covariance Matrix (SCM) has been widely used and this non-parametric estimator may be improved by exploiting the CCM structure. Toeplitz structure has been addressed by Burg in [1] while Fuhrmann in [2] used this estimator for radar detection purposes. In radar systems using a symmetrically spaced linear array with constant pulse repetition interval, the CCM has the persymmetric property. This structure information could then be exploited to improve detection performance. In this context, we use a particular linear transformation in order to take into account the persymmetry of the CCM and to study the statistical property of new detectors for both Gaussian and non-Gaussian environments. For Gaussian data, the CCM Maximum Likelihood (ML) estimator has been derived in [3]. The corresponding Generalized Likelihood Ratio Test (GLRT) has been investigated in [4]. For non Gaussian clutter modeled by Spherically Invariant Random Vectors (SIRV), detection schemes have been proposed in [5] and [6]. In [5] the persymmetry is only exploited to build two sets of independent data in order to derive a SIRV-Constant False Alarm Rate (CFAR) detector: the Persymmetric Adaptive Normalized Matched Filter (P-ANMF). In [6] these sets are used to initialize an iterative algorithm simultaneously proposed in [7] and [8]. This allows the derivation of the Recursive P-ANMF (RP-ANMF). Our approach, based on the Fixed Point Adaptive Normalized Matched Filter (FP-ANMF), also called GLRT-FP [7], [8], exploits an original transformation already proposed in [9] for Gaussian case and in [10] for non-Gaussian case. This leads to the Persymmetric Fixed Point Adaptive Normalized Matched Filter (PFP-ANMF) also called GLRT-PFP, i.e. the persymmetry property of the CCM. Its interest is to render the CCM real, leading to a simpler problem. Moreover this approach allows the derivation of the statistical analysis of the proposed detection scheme.

This paper is organized as follows. Section II presents the studied problem in terms of matrix estimation and radar detection and introduces persymmetry tools where it is shown how the persymmetric structure of the CCM can be exploited to provide the new Persymmetric Adaptive Matched Filter (PS-AMF). In section III, we derive the statistical distribution of the PS-AMF under hypothesis $H_{0}$, in which only noise is present in order to determine the improvement in terms of Probability of False Alarm (PFA). To confirm this improvement, some Gaussian data are extracted from the experimental data to validate the study. Section III also presents similarly the problem in non-Gaussian noise. The purpose is to derive an estimator of the CCM based on the secondary data and to take into account its structure (Persymmetric Fixed Point, $\left.\widehat{\mathbf{M}}_{P F P}\right)$. The statistical properties of $\widehat{\mathbf{M}}_{P F P}$ are also established and enable the investigation of the distribution of the detector $\Lambda_{G L R T-P F P}$, called GLRT-PFP (Generalized Likelihood Ratio Test - Persymmetric Fixed Point). Finally we present in section IV some simulated and experimental results 
that illustrate the improvement in terms of detection performance of the PS-AMF with respect to the conventional Adaptive Matched Filter (AMF) for Gaussian case. Moreover, results obtained with nonGaussian real data demonstrate the interest of the proposed detection scheme compared to the existing detectors P-ANMF, RP-ANMF and GLRT-FP.

\section{BACKGROUND AND PROBLEM STATEMENT}

This paragraph provides a guide to the notation used throughout the remainder of the paper. In general, a boldface, lowercase variable indicates a column vector quantity; a boldface, upper variable indicates a matrix; a variable with a caret $\left({ }^{\wedge}\right)$ is an estimate of an unknown quantity. Superscripts ${ }^{\top}$ or ${ }^{H}$ applied to a vector or a matrix denote the transpose or Hermitian (conjugate) transpose operations. The superscript

* denotes the conjugate operation. $E[$.] stands for the statistical expectation operator, $\operatorname{Tr}($.$) denotes the$ trace operator, $\mathcal{R} e($.$) denotes the real part and |$.$| denotes the determinant. In this paper, \|$. $\|$ stands for the usual $\mathcal{L}^{2}$-norm. $\mathbf{I}_{m}$ is the $m$-th order identity matrix, $\operatorname{Pr}($.$) stands for the probability and the notation$ $\sim$ means " is distributed as".

In radar detection, the main problem consists in detecting a signal $A \mathbf{p} \in \mathbb{C}^{m}$ corrupted by an additive clutter c. This problem can be stated as the following binary hypothesis test:

$$
\begin{cases}H_{0}: \mathbf{y}=\mathbf{c}, & \mathbf{y}_{\mathbf{k}}=\mathbf{c}_{\mathbf{k}}, \text { for } \mathbf{1} \leq \mathbf{k} \leq \mathbf{K}, \\ H_{1}: \mathbf{y}=\mathbf{A} \mathbf{p}+\mathbf{c}, & \mathbf{y}_{\mathbf{k}}=\mathbf{c}_{\mathbf{k}}, \text { for } \mathbf{1} \leq \mathbf{k} \leq \mathbf{K},\end{cases}
$$

where $\mathbf{y}$ is the complex $m$-vector of the received signal, $A$ is an unknown complex target amplitude and p stands for the known "steering vector". Under both hypotheses, it is assumed that $K$ signal-free data $\mathbf{y}_{\mathbf{k}}$ are available for clutter parameters estimation. The $\mathbf{y}_{\mathbf{k}}$ 's are the so-called secondary data where they are assumed independent, but their statistical distribution depends on the clutter nature. In this paper two cases will be investigated according to the clutter statistics: the Gaussian clutter and the case of non-Gaussian clutter as modeled by SIRV.

\section{A. Gaussian clutter}

In the Gaussian case, $\mathbf{c}$ and $\mathbf{c}_{k}$ are complex circular zero-mean Gaussian $m$-vectors sharing the same covariance matrix $\mathbf{M}$, with distribution denoted by $\mathcal{C N}(\mathbf{0}, \mathbf{M})$. When $\mathbf{M}$ is known, the GLRT for $A$ unknown is referred to as the Optimum Gaussian Detector (OGD):

$$
\Lambda_{O G D}=\frac{\left|\mathbf{p}^{H} \mathbf{M}^{-1} \mathbf{y}\right|^{2}}{\mathbf{p}^{H} \mathbf{M}^{-1} \mathbf{p}} \underset{H_{0}}{\gtrless} \lambda_{O G D},
$$


where the detection threshold $\lambda_{O G D}$ is related to the PFA $P_{f a}$ by $\lambda_{O G D}=-\ln \left(P_{f a}\right)$. However, in practice, the CCM $\mathbf{M}$ is generally unknown. One solution is to substitute for $\mathbf{M}$ an estimator $\widehat{\mathbf{M}}$ based on the secondary data. When no prior information on the M-structure is available, the SCM is classically used:

$$
\widehat{\mathbf{M}}_{S C M}=\frac{1}{K} \sum_{k=1}^{K} \mathbf{y}_{k} \mathbf{y}_{k}^{H} .
$$

leading to the so-called Adaptive Matched Filter (AMF) test [11]:

$$
\Lambda_{A M F}=\frac{\left|\mathbf{p}^{H} \widehat{\mathbf{M}}_{S C M}^{-1} \mathbf{y}\right|^{2}}{\mathbf{p}^{H} \widehat{\mathbf{M}}_{S C M}^{-1} \mathbf{p}} \underset{H_{0}}{\stackrel{H_{1}}{\gtrless}} \lambda_{A M F} .
$$

The relationship between the PFA $P_{f a}$ and the detection threshold $\lambda_{A M F}$ is given by [11], [12]:

$$
P_{f a}={ }_{2} F_{1}\left(K-m+1, K-m+2 ; K+1 ;-\frac{\lambda_{A M F}}{K}\right),
$$

where ${ }_{2} F_{1}($.$) is the hypergeometric function [13] defined by$

$$
{ }_{2} F_{1}(a, b ; c ; z)=\frac{\Gamma(c)}{\Gamma(b) \Gamma(c-b)} \int_{0}^{1} \frac{t^{b-1}(1-t)^{c-b-1}}{(1-t z)^{a}} d t .
$$

\section{B. Non-Gaussian clutter}

In recent years, there has been an increasing interest for non-Gaussian clutter models motivated by experimental radar clutter measurements [14], which have shown that the clutter is perfectly modeled by K-distribution or Weibull distribution. More generally, c can be modeled by a SIRV [15], [16] which is the product of the square root of a positive random variable $\tau$ (called the texture) and a $m$-dimensional independent complex Gaussian vector $\mathbf{g}$ (called the speckle) with zero-mean and covariance matrix $\mathbf{M}$ normalized according to $\operatorname{Tr}(\mathbf{M})=m$ for identifiability considerations [17]:

$$
\mathbf{c}=\sqrt{\tau} \mathbf{g} .
$$

The model when $\mathbf{M}$ is known and texture $\tau$ is unknown has been widely studied and this enables the construction of the Generalized Likelihood Ratio Test - Linear Quadratic (GLRT-LQ) [18], [17] defined by:

$$
\Lambda_{G L R T-L Q}=\frac{\left|\mathbf{p}^{H} \mathbf{M}^{-1} \mathbf{y}\right|^{2}}{\left(\mathbf{p}^{H} \mathbf{M}^{-1} \mathbf{p}\right)\left(\mathbf{y}^{H} \mathbf{M}^{-1} \mathbf{y}\right)} \underset{H_{0}}{\stackrel{H_{1}}{\gtrless}} \lambda_{G L R T-L Q},
$$

where $\lambda_{G L R T-L Q}$ is the corresponding detection threshold.

When $\mathbf{M}$ is unknown, one solution is to substitute a given estimator $\widehat{\mathbf{M}}$ of $\mathbf{M}$ in Eqn. (7) resulting in an adaptive version of the GLRT. When replacing $\mathbf{M}$ by an estimator $\widehat{\mathbf{M}}$, this detector is often called 
Adaptive Cosine Estimate (ACE) [19] or Adaptive Normalized Matched Filter (ANMF)

In non-Gaussian case, the CCM estimator $\widehat{\mathbf{M}}$ is based on the signal-free secondary data $\mathbf{y}_{k}=\mathbf{c}_{k}$ where the clutter samples $\mathbf{c}_{k}$ are SIRVs sharing the same CCM as $\mathbf{c}: \mathbf{c}_{k}=\sqrt{\tau_{k}} \mathbf{g}_{k}$, where $E\left[\mathbf{g}_{k} \mathbf{g}_{k}^{H}\right]=\mathbf{M}$. For the case when no prior information on the $\mathbf{M}$-structure is available, Conte and Gini in [7], [8] proposed an Approximate Maximum Likelihood (AML) estimator $\widehat{\mathbf{M}}_{F P}$ of $\mathbf{M}$, called the Fixed Point (FP) estimator, which is defined as the solution of the following implicit equation:

$$
\widehat{\mathbf{M}}_{F P}=\frac{m}{K} \sum_{k=1}^{K} \frac{\mathbf{y}_{k} \mathbf{y}_{k}^{H}}{\mathbf{y}_{k}^{H} \widehat{\mathbf{M}}_{F P}^{-1} \mathbf{y}_{k}} \text {. }
$$

Existence and uniqueness of the above equation solution have been proven in [20], while the complete statistical properties of $\widehat{\mathbf{M}}_{F P}$ have been derived in [21]. The estimator $\widehat{\mathbf{M}}_{F P}$ does not depend on the texture and allows to obtain the following adaptive detector GLRT-FP:

$$
\Lambda_{G L R T-F P}=\frac{\left|\mathbf{p}^{H} \widehat{\mathbf{M}}_{F P}^{-1} \mathbf{y}\right|^{2}}{\left(\mathbf{p}^{H} \widehat{\mathbf{M}}_{F P}^{-1} \mathbf{p}\right)\left(\mathbf{y}^{H} \widehat{\mathbf{M}}_{F P}^{-1} \mathbf{y}\right)}{\stackrel{H_{0}}{H_{1}}}_{\gtrless}^{\gtrless} \lambda_{G L R T-F P .}
$$

The relationship between the PFA $P_{f a}$ and the detection threshold $\lambda_{G L R T-F P}$ is given by [22]:

$$
P_{f a}=\left(1-\lambda_{G L R T-F P}\right)^{a-1}{ }_{2} F_{1}\left(a, a-1 ; b-1 ; \lambda_{G L R T-F P}\right) .
$$

where $K^{\prime}=\frac{m}{m+1} K, a=K^{\prime}-m+2$ and $b=K^{\prime}+2$.

\section{Persymmetry considerations and problem formulation}

It is clear that the estimation accuracy of $\widehat{\mathbf{M}}$ has an important impact on the adaptive detection performance in both Gaussian and non-Gaussian clutter. $\widehat{\mathbf{M}}_{S C M}$ and $\widehat{\mathbf{M}}_{F P}$ defined by Eqn. (3) and Eqn. (8) do not take into account any prior information on the CCM structure. However many applications lead to a CCM which exhibits some particular structure, and considering this structure may lead to a improvement in both estimation and detection performance. Such a situation is frequently met in radar systems using a symmetrically spaced linear array and a symmetrically spaced pulse train for temporal domain processing [1], [4], [5]. In these systems, the CCM M has the persymmetric property, defined as follows:

$$
\mathbf{M}=\mathbf{J}_{m} \mathbf{M}^{*} \mathbf{J}_{m},
$$


where $\mathbf{J}_{m}$ is the $m$-dimensional antidiagonal matrix having 1 as non-zero elements. The steering vector of the problem is also persymmetric, i.e. it satisfies:

$$
\mathbf{p}=\mathbf{J}_{m} \mathbf{p}^{*}
$$

The persymmetric structure of $\mathbf{M}$ will be exploited in this paper in order to improve its estimation accuracy compared to unstructured estimators. This will be done by means of the transformation matrix $\mathbf{T}$ introduced in [23] and whose properties are recalled in the following proposition.

Proposition II.1 ( [23] ) Let $\boldsymbol{T}$ be the unitary matrix defined as:

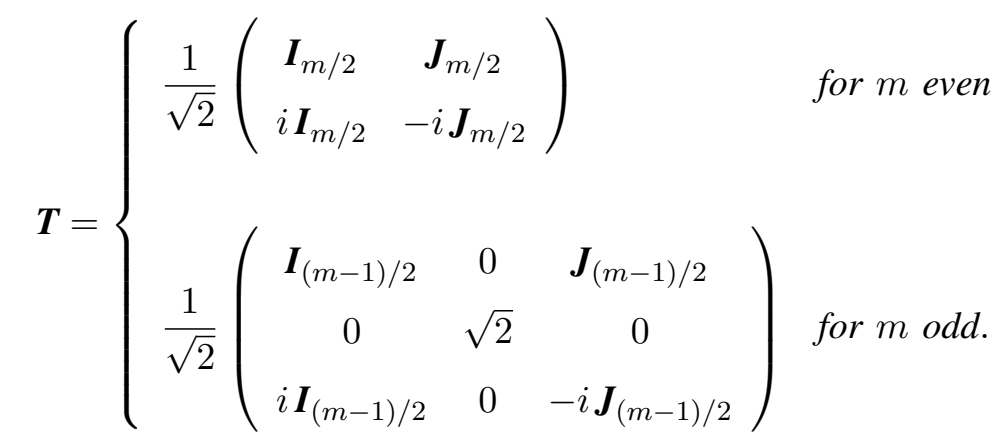

Persymmetric vectors and Hermitian matrices are characterized by the following properties:

- $\boldsymbol{p} \in \mathbb{C}^{m}$ is a persymmetric vector if and only if $\boldsymbol{T} \boldsymbol{p}$ is a real vector.

- $\boldsymbol{M}$ is a persymmetric Hermitian matrix if and only if $\boldsymbol{T} \boldsymbol{M} \boldsymbol{T}^{H}$ is a real symmetric matrix.

Using proposition II.1, the original problem (1) can be equivalently reformulated. Let us introduce the transformed primary data $\mathbf{x}$, the transformed secondary data $\mathbf{x}_{k}$, the transformed clutter vector $\mathbf{n}$ and the transformed signal steering vector $\mathbf{s}$ defined as: $\mathbf{x}=\mathbf{T} \mathbf{y}, \mathbf{x}_{k}=\mathbf{T} \mathbf{y}_{k}, \mathbf{s}=\mathbf{T} \mathbf{p}, \mathbf{n}=\mathbf{T} \mathbf{c}, \mathbf{n}_{k}=\mathbf{T} \mathbf{c}_{k}$.

It follows that the transformed signal steering vector $\mathbf{s}$ and the transformed CCM are both real. Then, the original problem (1) is equivalent to:

$$
\begin{cases}H_{0}: \mathbf{x}=\mathbf{n} & \mathbf{x}_{\mathbf{k}}=\mathbf{n}_{\mathbf{k}}, \text { for } \mathbf{1} \leq \mathbf{k} \leq \mathbf{K}, \\ H_{1}: \mathbf{x}=\mathbf{A} \mathbf{s}+\mathbf{n} & \mathbf{x}_{\mathbf{k}}=\mathbf{n}_{\mathbf{k}}, \text { for } \mathbf{1} \leq \mathbf{k} \leq \mathbf{K},\end{cases}
$$

where $\mathbf{x} \in \mathbb{C}^{m}, \mathbf{s}$ is a known real vector.

In the Gaussian case, under hypothesis $H_{0}, \mathbf{n}$ and the $K$ transformed secondary data $\mathbf{x}_{k}$ are i.i.d and share the same $\mathcal{C N}(\mathbf{0}, \mathbf{R})$ distribution where $\mathbf{R}=\mathbf{T} \mathbf{M} \mathbf{T}^{H}$ is a real symmetric matrix according to proposition II.1. 
In the Non-Gaussian case, one has:

$$
\begin{array}{r}
\mathbf{n}=\sqrt{\tau} \mathbf{h} \\
\mathbf{n}_{k}=\sqrt{\tau_{k}} \mathbf{h}_{k}
\end{array}
$$

where $\mathbf{h}=\mathbf{T} \mathbf{g}$ and $\mathbf{h}_{k}=\mathbf{T} \mathbf{g}_{k}$ denote the transformed speckle vector with the same real covariance

matrix $\mathbf{R}=\mathbf{T} \mathbf{M} \mathbf{T}^{H}$. $\mathbf{n}$ and $\mathbf{n}_{k}$ are still SIRV's with the same texture and CCM $\mathbf{R}=\mathbf{T} \mathbf{M} \mathbf{T}^{H}$. From now on, the problem under study is the problem defined by (14).

\section{Detection Scheme}

In this section the detection problem (14) is investigated in Gaussian and non-Gaussian frameworks. More precisely, the CCM estimation problem is addressed and adaptive detection schemes are proposed. In both cases, the adaptive detector properties are studied.

\section{A. Detection in circular Gaussian noise}

Let us first investigate the ML estimator of the real covariance matrix $\mathbf{R}$ from the $K$ secondary data $\mathbf{x}_{k}$. The main motivation for introducing the transformed data is that the resulting distribution of the ML estimator of $\mathbf{R}$ is very simple. This was not the case in [3] when dealing with the original secondary data $\mathbf{y}_{k}$ with persymmetric covariance matrix.

Proposition III.1 The ML estimator $\widehat{\boldsymbol{R}}_{P}$ of the real matrix $\boldsymbol{R}$ is unbiased and is given by:

$$
\widehat{\boldsymbol{R}}_{P}=\mathcal{R} e\left(\widehat{\boldsymbol{R}}_{S C M}\right),
$$

where:

$$
\widehat{\boldsymbol{R}}_{S C M}=\frac{1}{K} \sum_{k=1}^{K} \boldsymbol{x}_{k} \boldsymbol{x}_{k}^{H}=\boldsymbol{T} \widehat{\boldsymbol{M}}_{S C M} \boldsymbol{T}^{H}
$$

$\widehat{\boldsymbol{R}}_{P}$ is an unbiased estimator and $K \widehat{\boldsymbol{R}}_{P}$ is real Wishart distributed with parameter matrix $\frac{1}{2} \boldsymbol{R}$ and $2 K$ degrees of freedom.

Proof: It is easy to show that the MLE of the real covariance matrix is provided by equation (17).

Let us now investigate its statistical properties. Let $\mathbf{a}_{k}$ and $\mathbf{b}_{k}$ be the real and imaginary parts of the secondary data:

$$
\mathbf{x}_{k}=\mathbf{a}_{k}+i \mathbf{b}_{k}
$$


and

$$
K \widehat{\mathbf{R}}_{P}=\sum_{k=1}^{K} \mathbf{a}_{k} \mathbf{a}_{k}^{\top}+\sum_{k=1}^{K} \mathbf{b}_{k} \mathbf{b}_{k}^{\top}
$$

$\mathbf{x}_{k}$ is circular, i.e. $E\left[\mathbf{x}_{k} \mathbf{x}_{k}^{\top}\right]=\mathbf{0}$ which leads to:

$$
\begin{aligned}
& E\left[\mathbf{a}_{k} \mathbf{a}_{k}^{\top}\right]-E\left[\mathbf{b}_{k} \mathbf{b}_{k}^{\top}\right]=\mathbf{0} \\
& E\left[\mathbf{a}_{k} \mathbf{b}_{k}^{\top}\right]+E\left[\mathbf{b}_{k} \mathbf{a}_{k}^{\top}\right]=\mathbf{0}
\end{aligned}
$$

Moreover, $\mathbf{x}_{k}$ has a real covariance matrix $\mathbf{R}$ which implies:

$$
\begin{aligned}
E\left[\mathbf{a}_{k} \mathbf{a}_{k}^{\top}\right]+E\left[\mathbf{b}_{k} \mathbf{b}_{k}^{\top}\right]=\mathbf{R} \\
E\left[\mathbf{a}_{k} \mathbf{b}_{k}^{\top}\right]-E\left[\mathbf{b}_{k} \mathbf{a}_{k}^{\top}\right]=\mathbf{0}
\end{aligned}
$$

Equations (21) and (22) yield:

$$
\begin{aligned}
E\left[\mathbf{a}_{k} \mathbf{a}_{k}^{\top}\right] & =E\left[\mathbf{b}_{k} \mathbf{b}_{k}^{\top}\right]=\frac{1}{2} \mathbf{R} \\
E\left[\mathbf{a}_{k} \mathbf{b}_{k}^{\top}\right] & =\mathbf{0}
\end{aligned}
$$

showing that the $\mathbf{a}_{k}$ 's and the $\mathbf{b}_{k}$ 's are independent and share the same covariance matrix $\frac{1}{2} \mathbf{R}$.

From Eqn. (20), $K \widehat{\mathbf{R}}_{P}$ has a real Wishart distribution with $2 K$ degrees of freedom and parameter matrix $\frac{1}{2} \mathbf{R}$. Moreover,

$$
E\left[K \widehat{\mathbf{R}}_{P}\right]=2 K \frac{1}{2} \mathbf{R}
$$

resulting in $E\left[\widehat{\mathbf{R}}_{P}\right]=\mathbf{R}$.

Actually, taking into account the real structure of $\mathbf{R}$ (or equivalently the persymmetric structure of $\mathbf{M}$ ) in the ML estimation procedure allows to virtually double the number of secondary data. Let us now consider the AMF for the detection problem (14) based on the estimator $\widehat{\mathbf{R}}_{P}$ defined by (17). This leads to the following detection test, called the PS-AMF,

$$
\Lambda_{P S-A M F}=\frac{\left|\mathbf{s}^{\top} \widehat{\mathbf{R}}_{P}^{-1} \mathbf{x}\right|^{2}}{\mathbf{s}^{\top} \widehat{\mathbf{R}}_{P}^{-1} \mathbf{S}} \underset{H_{0}}{\stackrel{H_{1}}{\gtrless}} \lambda_{P S-A M F},
$$

or equivalently, in terms of the original data,

$$
\Lambda_{P S-A M F}=\frac{\left|\mathbf{p}^{H} \mathbf{T}^{H}\left[\mathcal{R} e\left(\mathbf{T} \widehat{\mathbf{M}}_{S C M} \mathbf{T}^{H}\right)\right]^{-1} \mathbf{T y}\right|^{2}}{\mathbf{p}^{H} \mathbf{T}^{H}\left[\mathcal{R} e\left(\mathbf{T} \widehat{\mathbf{M}}_{S C M} \mathbf{T}^{H}\right)\right]^{-1} \mathbf{T} \mathbf{p}}{\underset{H}{H_{0}}}_{H_{1}}^{\gtrless} \lambda_{P S-A M F} .
$$


The distribution of Eqn. (25) is well-known when $K \widehat{\mathbf{R}}_{P}$ is complex Wishart distributed with parameter matrix $K \mathbf{R}$ and $K$ degrees of freedom: this is the classical AMF distribution [11], [24]. However, in our problem, $K \widehat{\mathbf{R}}_{P}$ is real Wishart distributed with parameter matrix $\frac{1}{2} \mathbf{R}$ and $2 K$ degrees of freedom while $\mathbf{x}$ is complex. The following proposition establishes the statistical distribution of the PS-AMF and the relationship between the PFA $P_{f a}$ and the detection threshold $\lambda_{P S-A M F}$.

Proposition III.2 • Under $H_{0}$, the PDF $p(z)$ of $\Lambda_{P S-A M F}$, defined by Eqn. (25), is:

$$
p(z)=\frac{(2 K-m+1)(2 K-m+2)}{2 K(2 K+1)}{ }_{2} F_{1}\left(\frac{2 K-m+3}{2}, \frac{2 K-m+4}{2} ; \frac{2 K+3}{2} ;-\frac{z}{K}\right),
$$

- The relationship between $P_{f a}$ and the detection threshold $\lambda$ is:

$$
P_{f a}={ }_{2} F_{1}\left(\frac{2 K-m+1}{2}, \frac{2 K-m+2}{2} ; \frac{2 K+1}{2} ;-\frac{\lambda_{P S-A M F}}{K}\right) .
$$

Proof: The proof is given on Appendix VII-A

As will be seen in section IV for both simulated and experimental data, the PS-AMF outperforms the AMF, especially for a small number of secondary data.

\section{B. Detection in non-Gaussian noise}

The purpose of this section is to address the non-Gaussian case for the detection problem (14). Let us first recall some notations. The additive SIRV noise $\mathbf{n}$ is defined by:

$$
\mathbf{n}=\sqrt{\tau} \mathbf{h}
$$

where $\tau$ is a positive random variable, and $\mathbf{h}$ is a zero-mean circular complex Gaussian vector with real CCM R. The $K$ secondary data $\mathbf{n}_{k}=\sqrt{\tau_{k}} \mathbf{h}_{k}$ are i.i.d. and share the same distribution as $\mathbf{n}$.

Since the transformed CCM $\mathbf{R}$ is real, its structure may be taken into account in the estimation procedure by retaining only the real part of the FP estimator. This leads to the proposed covariance estimator called the Persymmetric Fixed-Point since it results from the persymmetric structure of the original speckle covariance matrix:

$$
\widehat{\mathbf{R}}_{P F P}=\mathcal{R} e\left(\widehat{\mathbf{R}}_{F P}\right),
$$

with

$$
\widehat{\mathbf{R}}_{F P}=\mathbf{T} \widehat{\mathbf{M}}_{F P} \mathbf{T}^{H} .
$$


In this section, the statistical properties of the detector $\Lambda_{G L R T-P F P}$ are investigated under the null hypothesis $H_{0}$. Let us recall some basic definitions:

- A test statistic is said to be texture-CFAR when its distribution is independent of the texture distribution,

- A test statistic is said to be matrix-CFAR when its distribution is independent of $\mathbf{R}$,

- A test statistic is said to be SIRV-CFAR when it is both texture-CFAR and matrix-CFAR.

The statistical properties of $\widehat{\mathbf{R}}_{P F P}$ are provided by the following proposition.

\section{Proposition III.3 (Statistical performance of $\widehat{\mathbf{R}}_{P F P}$ )}

- The distribution of $\widehat{\boldsymbol{R}}_{P F P}$ does not depend on the texture.

- $\widehat{\boldsymbol{R}}_{P F P}$ is a consistent estimator of $\boldsymbol{R}$.

- $\widehat{\boldsymbol{R}}_{P F P}$ is an unbiased estimator of $\boldsymbol{R}$.

- $\frac{\widehat{\boldsymbol{R}}_{P F P}}{\operatorname{Tr}\left(\boldsymbol{R}^{-1} \widehat{\boldsymbol{R}}_{P F P}\right)}$ has the same asymptotic distribution as $\frac{\widehat{\boldsymbol{R}}}{\operatorname{Tr}\left(\boldsymbol{R}^{-1} \widehat{\boldsymbol{R}}\right)}$, where $\widehat{\boldsymbol{R}}$ is real Wishart distributed with $\frac{m}{m+1} 2 K$ degrees of freedom and parameter matrix $\boldsymbol{R}$.

Proof: In [21], unbiasedness and consistency of $\widehat{\mathbf{R}}_{F P}$ are proved. Taking the real part of this estimator does not change these two statistical properties.

It has been shown in [21] that $\frac{\widehat{\mathbf{R}}_{F P}}{\operatorname{Tr}\left(\mathbf{R}^{-1} \widehat{\mathbf{R}}_{F P}\right)}$ has the same asymptotic distribution as $\frac{\widehat{\mathbf{R}}_{c}}{\operatorname{Tr}\left(\mathbf{R}^{-1} \widehat{\mathbf{R}}_{c}\right)}$ with $\widehat{\mathbf{R}}_{c}$ complex Wishart distributed with $\frac{m}{m+1} K$ degrees of freedom and parameter matrix $\mathbf{R}$. Therefore $\frac{\widehat{\mathbf{R}}_{P F P}}{\operatorname{Tr}\left(\mathbf{R}^{-1} \widehat{\mathbf{R}}_{P F P}\right)}$ has the same asymptotic distribution as $\frac{\mathcal{R} e\left(\widehat{\mathbf{R}}_{F P}\right)}{\operatorname{Tr}\left(\mathbf{R}^{-1} \mathcal{R} e\left(\widehat{\mathbf{R}}_{F P}\right)\right)}$. By noting that $\operatorname{Tr}(\mathbf{A B})=$ $\operatorname{Tr}(\mathbf{A} \mathcal{R} e(\mathbf{B}))$ when $\mathbf{A}$ is real symmetric and $\mathbf{B}$ is Hermitian, it follows that $\frac{\widehat{\mathbf{R}}_{P F P}}{\operatorname{Tr}\left(\mathbf{R}^{-1} \widehat{\mathbf{R}}_{P F P}\right)}$ has the same asymptotic distribution as $\frac{\widehat{\mathbf{R}}}{\operatorname{Tr}\left(\mathbf{R}^{-1} \widehat{\mathbf{R}}\right)}$, where $\widehat{\mathbf{R}}=2 \mathcal{R} e\left(\mathbf{R}_{c}\right)$ is real Wishart distributed with parameter matrix $\mathbf{R}$ and $K^{\prime}=\frac{m}{m+1} 2 K$ degrees of freedom.

Definition III.1 The adaptive GLRT, for the transformed problem (14), based on Eqn. (7) and on the PFP estimator is:

$$
\Lambda_{G L R T-P F P}=\frac{\left|\boldsymbol{s}^{\top} \widehat{\boldsymbol{R}}_{P F P}^{-1} \boldsymbol{x}\right|^{2}}{\left(\boldsymbol{s}^{\top} \widehat{\boldsymbol{R}}_{P F P}^{-1} \boldsymbol{s}\right)\left(\boldsymbol{x}^{H} \widehat{\boldsymbol{R}}_{P F P}^{-1} \boldsymbol{x}\right)}{\underset{H}{H_{0}}}_{H_{1}}^{\gtrless} \lambda_{G L R T-P F P} .
$$


Proposition III.4 $\Lambda_{G L R T-P F P}$ is SIRV-CFAR. For large $K$, under hypothesis $H_{0}, \Lambda_{G L R T-P F P}$ has the same distribution as $\Lambda=\frac{\left|\boldsymbol{e}_{1}^{\top} \widehat{\boldsymbol{W}}^{-1} \boldsymbol{w}\right|^{2}}{\left(\boldsymbol{e}_{1}^{\top} \widehat{\boldsymbol{W}}^{-1} \boldsymbol{e}_{1}\right)\left(\boldsymbol{w}^{H} \widehat{\boldsymbol{W}}^{-1} \boldsymbol{w}\right)}$ where $\boldsymbol{w} \sim \mathcal{C N}(0, \boldsymbol{I}), \boldsymbol{e}_{1}=(1,0, \ldots, 0)^{\top}$ and where $\widehat{\boldsymbol{W}}$ is real Wishart distributed with parameter matrix $\boldsymbol{I}$ and $K^{\prime}=\frac{m}{m+1} 2 K$ degrees of freedom.

Proof: Since the FP estimator $\widehat{\mathbf{M}}_{F P}$ does not depend on the texture, it follows from Eqn. (29) and (30) that $\widehat{\mathbf{R}}_{P F P}$ is itself texture independent. Moreover, under hypothesis $H_{0}, \Lambda_{G L R T-P F P}$ is homogeneous in terms of $\tau$. Therefore, $\Lambda_{G L R T-P F P}$ is texture-CFAR.

Let us now investigate the matrix-CFAR property. Let $\mathbf{R}^{\frac{1}{2}} \mathbf{R}^{\frac{T}{2}}$ be a real factorization of $\mathbf{R}$, and let $\mathbf{Q}$ be a real unitary matrix such that:

$$
\mathrm{QR}^{-1 / 2} \mathrm{~s}=\left(\mathbf{s}^{\top} \mathbf{R}^{-1} \mathrm{~s}\right)^{1 / 2} \mathbf{e}_{1}
$$

Note that the last equation is possible with $\mathbf{Q}$ real since $\mathbf{s}$ is itself real.

The test statistic $\Lambda_{G L R T-P F P}$ may then be rewritten

$$
\Lambda_{G L R T-P F P}=\frac{\left|\mathbf{e}_{1}^{\top} \widehat{\mathbf{A}}^{-1} \mathbf{w}\right|^{2}}{\left(\mathbf{e}_{1}^{\top} \widehat{\mathbf{A}}^{-1} \mathbf{e}_{1}\right)\left(\mathbf{w}^{H} \widehat{\mathbf{A}}^{-1} \mathbf{w}\right)}
$$

where

$$
\mathbf{w}=\mathbf{Q} \mathbf{R}^{-\frac{1}{2}} \mathbf{h} \sim \mathcal{C N}(\mathbf{0}, \mathbf{I})
$$

and where

$$
\begin{aligned}
\widehat{\mathbf{A}} & =\mathbf{Q} \mathbf{R}^{-\frac{1}{2}} \widehat{\mathbf{R}}_{P F P} \mathbf{R}^{-\frac{\tau}{2}} \mathbf{Q}^{\top} \\
& =\mathcal{R} e\left(\mathbf{Q} \mathbf{R}^{-\frac{1}{2}} \widehat{\mathbf{R}}_{F P} \mathbf{R}^{-\frac{\tau}{2}} \mathbf{Q}^{\top}\right)
\end{aligned}
$$

It has been shown in [25] that $\mathbf{Q} \mathbf{R}^{-\frac{1}{2}} \widehat{\mathbf{R}}_{F P} \mathbf{R}^{-\frac{T}{2}} \mathbf{Q}^{\top}$ in Eqn. (35) is a FP estimator of the identity matrix and that its distribution is therefore independent of $\mathbf{R}$. Thus, the same conclusion holds for its real part $\widehat{\mathbf{A}}$ defined by Eqn. (35) and the matrix-CFAR property is proved.

From the fourth point of proposition III.3, $\Lambda_{G L R T-P F P}$ has the same distribution as

$$
\Lambda=\frac{\left|\mathbf{s}^{\top} \widehat{\mathbf{R}}^{-1} \mathbf{x}\right|^{2}}{\left(\mathbf{s}^{\top} \widehat{\mathbf{R}}^{-1} \mathbf{s}\right)\left(\mathbf{x}^{H} \widehat{\mathbf{R}}^{-1} \mathbf{x}\right)},
$$

where $\widehat{\mathbf{R}}$ is real Wishart distributed with $K^{\prime}$ degrees of freedom and parameter matrix $\mathbf{R}$. 
Let $\mathbf{Q}$ be the real unitary matrix defined by Eqn. (32) and let us define $\widehat{\mathbf{W}}=\mathbf{Q} \mathbf{R}^{-\frac{1}{2}} \widehat{\mathbf{R}} \mathbf{R}^{-\frac{T}{2}} \mathbf{Q}^{\top}$. The matrix $\widehat{\mathbf{W}}$ is real Wishart distributed with $K^{\prime}$ degrees of freedom and parameter matrix I. Then $\Lambda$ defined by Eqn. (36) can be rewritten as

$$
\Lambda=\frac{\left|\mathbf{e}_{1}^{\top} \widehat{\mathbf{W}}^{-1} \mathbf{w}\right|^{2}}{\left(\mathbf{e}_{1}^{\top} \widehat{\mathbf{W}}^{-1} \mathbf{e}_{1}\right)\left(\mathbf{w}^{H} \widehat{\mathbf{W}}^{-1} \mathbf{w}\right)},
$$

which concludes the proof.

Moreover, in the sequel, all the statistical properties can be analyzed by choosing $\mathbf{n}$ and $\mathbf{n}_{k}$ to be Gaussian distributed because of these texture-CFAR properties. The analytical expression for the PDF of the detector $\Lambda_{G L R T-P F P}$ has not been derived but the following theorem gives some insight about its distribution. This derivation is important from an operational point of view in order to regulate the false alarm.

Proposition III.5 For large $K, \Lambda_{G L R T-P F P}$ has the same distribution as $\frac{F}{F+1}$ where:

$$
F=\frac{\left(\alpha_{1} l_{22}-\alpha_{2} l_{21}\right)^{2}+\left(1+\left(\frac{\beta_{3}}{l_{33}}\right)^{2}\right)\left(a l_{22}-b l_{21}\right)^{2}}{\left(\alpha_{2} l_{11}\right)^{2}+\left(l_{11} l_{22} \frac{\beta_{3}}{l_{33}}\right)^{2}+l_{11}^{2}\left(1+\left(\frac{\beta_{3}}{l_{33}}\right)^{2}\right) b^{2}}
$$

and where all the following random variables are independent and distributed according to:

$$
a, b, \alpha_{1}, l_{21} \sim \mathcal{N}(0,1), \alpha_{2}^{2} \sim \chi_{m-1}^{2}, \beta_{3}^{2} \sim \chi_{m-2}^{2}, l_{11}^{2} \sim \chi_{K^{\prime}-m+1}^{2}, l_{22}^{2} \sim \chi_{K^{\prime}-m+2}^{2}, l_{33}^{2} \sim \chi_{K^{\prime}-m+3}^{2},
$$

with $K^{\prime}=\frac{m}{m+1} 2 K$.

Proof: The proof of this proposition is given on Appendix VII-B.

Proposition III.5 may be used to obtain, through Monte-Carlo simulations, the relation between the PFA and the detection threshold $\lambda$ for the GRLT-PFP (31).

\section{VALIDATION ON EXPERIMENTAL DATA}

After the statistical study of these detectors, this section presents some results obtained on some experimental and simulated data in Gaussian and non-Gaussian case. 


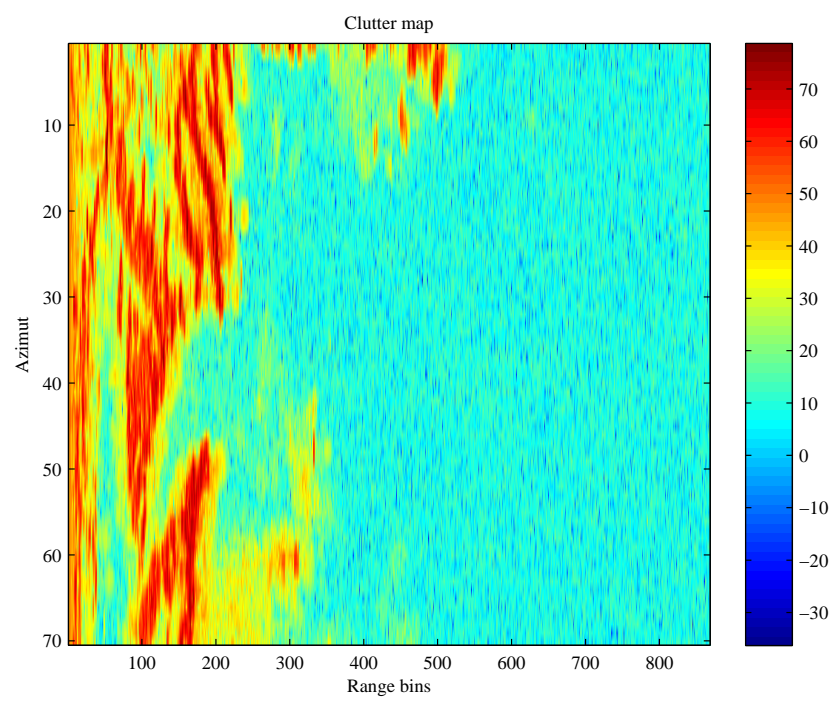

Fig. 1. Ground clutter data level (in $\mathrm{dB}$ ) corresponding to the first pulse.

\section{A. Experimental Gaussian data extraction and validation}

The ground clutter data used in this paper were collected by an operational radar at Thales Air System. The radar is 13 meters above the ground and illuminating the ground at low grazing angle. Ground clutter complex echoes were collected for $N=868$ range bins, for 70 different azimuth angles and for $m=8$ pulses. Fig. 1 displays the ground clutter data level (in $\mathrm{dB}$ ) corresponding to the first pulse echo. Near the radar, echoes characterize heterogeneous ground clutter whereas beyond the radioelectric horizon of the radar (around $15 \mathrm{~km}$ ), only heterogeneous thermal noise (the blue part of the map) is present.

In order to test the PS-AMF and given the non-Gaussian nature of experimental data, it is necessary to select particular data on the radar map. In addition, on some operational map, further parts of the data present a low amplitude. Beyond the electromagnetic horizon of the radar, the absence of reflectors gives an homogeneous area of data, characterized as Gaussian thermal noise. A statistical selection of these data allows us to obtain experimental Gaussian noise to test our detection algorithm.

For that purpose, the well-known goodness-of-fit test of Kolmogorov-Smirnov (KS) is widely used [26]. This test verifies the adequacy of a given data sample to a Gaussian distributed sample $\mathcal{N}(\mu ; \sigma)$. In order to obtain an homogeneous area of data, the KS test is applied successively on little blocks of data. With this test, the non-Gaussian data are rejected but the obtained map is lacunar. Indeed, the original 
clutter map presents a particular structure where there are horizontal band corresponding to constant azimuths of the radar. The transitions between these bands show a significant difference of the mean of the data and a consequence is that the KS test is then inefficient. This is also the case for another goodness-of-fit test like Anderson-Darling [26].

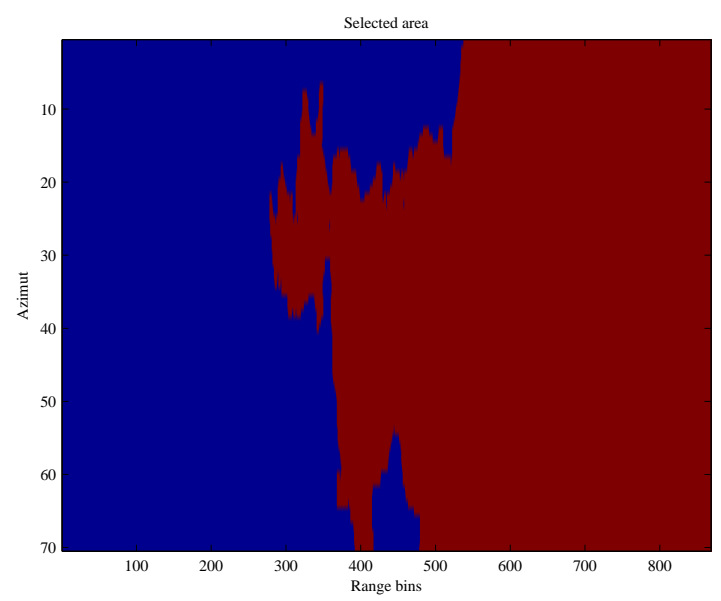

(a) Binary area selected for Gaussian data extraction (Gaussian area in red and non-Gaussian in blue).

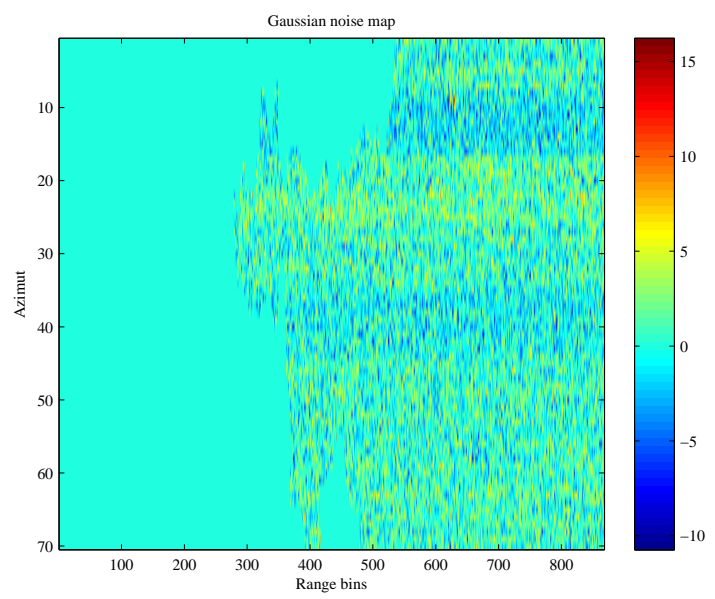

(b) Gaussian map (in $\mathrm{dB}$ ) corresponding to the Gaussian selection and extracted from entire map

Fig. 2. Selection of the Gaussian area in the data presented in Fig. 1

In order to get round this problem, a robust algorithm using connectedness of the data is developed. 
The idea is to include in the Gaussian area all the little blocks of supposed non-Gaussian data when they are not connected with the main non-Gaussian area. All the little blocks of data which are Gaussian but not characterized as Gaussian because of the non homogeneity of the KS test are now included in the main Gaussian area. We obtain with this method a wide area of Gaussian homogeneous data. In Fig. 2(a), we present the Gaussian area selected by the statistical study of the entire map. Gaussian area is colored in red and non-Gaussian area is in blue. This map confirms that only Gaussian thermal noise is present on the radioelectric horizon of the radar. In terms of experimental data, we present on Fig. 2(b) the clutter map with only Gaussian data extracted. The similar color map as in Fig. 1 allows to verify the adequacy between original and extracted data. 


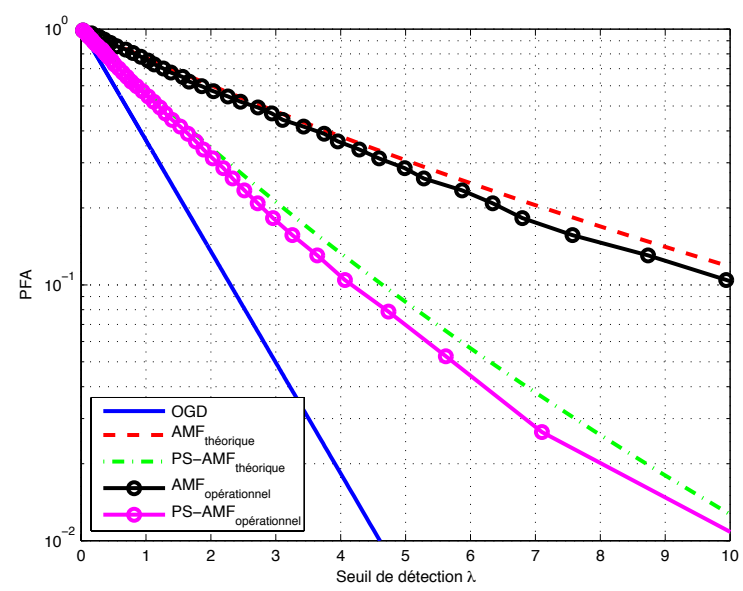

(a) Theoretical and experimental PFA-threshold curves for various Gaussian detectors.

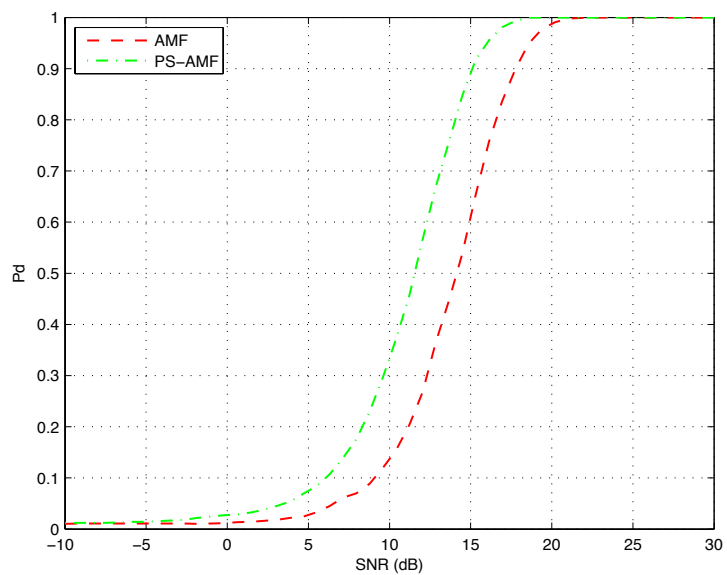

(b) Probability of Detection versus SNR for the AMF and PS-AMF

Fig. 3. Comparison between Performance of detectors for $P_{f a}=10^{-2}, m=8$ and $K=14$.

Once Gaussian data have been extracted from entire clutter map, we present the classical performance of this detector. In Fig. 3(a) and Fig. 4(a), the PFA with respect to detection threshold are presented. The theoretical relations for the OGD, the AMF and the PS-AMF are compared to the experimental relation (AMF and PS-AMF) in order to confirm the validity of the statistical study. Experimental curves are determined by Monte-Carlo counting, moving a $(5 \times 3)$ and $(5 \times 5)$ CFAR mask with different number of simulations (nsimu). The theoretical relation is then validated. In Fig. 3(b) and 4(b), we present the Probability of Detection versus SNR in order to verify and to quantify the improvement in terms of detection for the PS-AMF compared to the AMF. However, these figures show the benefit of taking into 
account the persymmetric structure of the CCM in the Gaussian case.

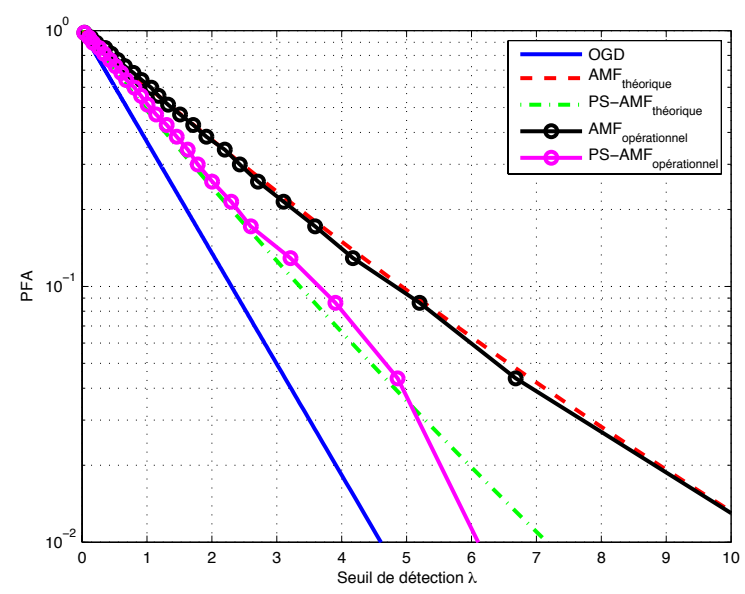

(a) Theoretical and experimental PFA-threshold curves for various Gaussian detectors.

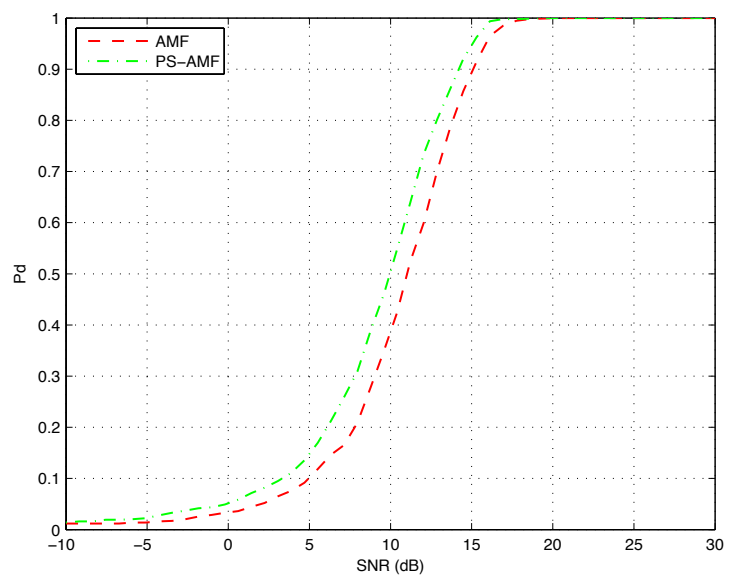

(b) Probability of Detection versus SNR for the AMF and PS-AMF

Fig. 4. Comparison between Performance of detectors for $P_{f a}=10^{-2}, m=8$ and $K=24$.

\section{B. Non-Gaussian experiments}

In the context of non-Gaussian clutter, Conte and De Maio in [5] and [6] have proposed two detectors derived respectively from the GLRT with some different estimators: the P-ANMF and the RP-ANMF. In [5], the persymmetry property is only used to separate their original set of secondary data $\mathbf{n}_{k}$ into two new uncorrelated and then independent sets of data $\mathbf{r}_{e k}$ and $\mathbf{r}_{o k}$, in order to render the detector matrix-CFAR and improve the performance in terms of detection. These new vectors have the same size 
as the original, and share the same texture. Their speckle components are i.i.d. and zero-mean complex Gaussian vectors. These news sets of secondary data allow the introduction of their new estimator for the CCM:

$$
\widehat{\boldsymbol{\Sigma}}=\frac{1}{K} \sum_{k=1}^{K} \frac{\mathbf{r}_{e k} \mathbf{r}_{e k}^{H}}{\left(\left|\mathbf{r}_{o k} \mathbf{r}_{o k}^{H}\right|\right)_{i, i}} .
$$

where $(\mathbf{A})_{i, i}$ stands for any $(i, i)$ th element of the matrix $\mathbf{A}$.

The previous estimator is then replaced in the classical GLRT-LQ given by (7) which leads to the P-ANMF detector defined as:

$$
\Lambda_{P-A N M F}=\frac{\left|\mathbf{p}^{H} \widehat{\boldsymbol{\Sigma}}^{-1} \mathbf{x}\right|^{2}}{\left(\mathbf{p}^{H} \widehat{\boldsymbol{\Sigma}}^{-1} \mathbf{p}\right)\left(\mathbf{x}^{H} \widehat{\boldsymbol{\Sigma}}^{-1} \mathbf{x}\right)} \underset{H_{0}}{\stackrel{H_{1}}{\gtrless}} \lambda_{P-A N M F} .
$$

In [6], the same method is used to define two set of secondary data $\mathbf{r}_{e k}$ and $\mathbf{r}_{o k}$ and the FP matrix estimator $\widehat{\boldsymbol{\Sigma}}_{(\mathrm{inf})}$ is found by using the recursive procedure:

$$
\widehat{\boldsymbol{\Sigma}}_{(t+1)}=\frac{N}{K} \sum_{k=1}^{K} \frac{\mathbf{r}_{e k} \mathbf{r}_{e k}^{H}}{\mathbf{r}_{e k}^{H}\left(\widehat{\boldsymbol{\Sigma}}_{(t)}\right)^{-1} \mathbf{r}_{e k}}
$$

with starting point:

$$
\widehat{\boldsymbol{\Sigma}}_{(0)}=\frac{1}{K} \sum_{1}^{K} \frac{\mathbf{r}_{e k} \mathbf{r}_{e k}^{H}}{\left(\left|\mathbf{T} \mathbf{r}_{o k} \mathbf{r}_{o k}^{H} \mathbf{T}^{H}\right|\right)_{i, i}}
$$

This estimator is next replaced in the GLRT-LQ (7) to provide the RP-ANMF:

$$
\Lambda_{R P-A N M F}=\frac{\left|\mathbf{p}^{H} \widehat{\mathbf{\Sigma}}_{(\mathrm{inf})}^{-1} \mathbf{x}\right|^{2}}{\left(\mathbf{p}^{H} \widehat{\boldsymbol{\Sigma}}_{(\mathrm{inf})}^{-1} \mathbf{p}\right)\left(\mathbf{x}^{H} \widehat{\mathbf{\Sigma}}_{(\mathrm{inf})}^{-1} \mathbf{x}\right)} \gtrless_{H_{0}}^{H_{1}} \lambda_{R P-A N M F} .
$$

Please note that, as stated in [20], the solution $\widehat{\mathbf{\Sigma}}^{(\mathrm{inf})}$ of the implicit FP matrix equation is unique and does not depend on the starting point.

In order to compare all these detectors (GLRT-LQ with M known or with the classical SCM, GLRT-FP, GLRT-PFP, P-ANMF and RP-ANMF), we present in Fig. 5(a) the PFA versus the detection threshold for all these detectors and in Fig. 5(b), the Probability of Detection versus the Signal to Noise Ratio (SNR). The simulated impulsive clutter is in this case chosen to be K-distributed [27]:

$$
f_{x}(x)=\frac{2}{a \Gamma(\nu+1)}\left(\frac{x}{2 a}\right)^{\nu+1} K_{\nu}\left(\frac{x}{a}\right)
$$

where $\Gamma($.$) is the standard Gamma function [13] and K_{\nu}$ is the modified Bessel function of order $\nu$ [13] and where $a$ and $\nu$ are constant positive parameters. 
These figures show the improvement in terms of detection of the RP-ANMF on the conventional GLRT-SCM (which is not efficient on non-Gaussian data) but also the improvement of the GLRT-PFP on all the other detectors. Moreover, theoretical results based on the asymptotic Wishart distributions of $\widehat{\mathbf{R}}_{F P}$ and $\widehat{\mathbf{R}}_{P F P}$ (circle lines) are displayed. It can be noticed that the simulated results are in very good agreement with the theory.

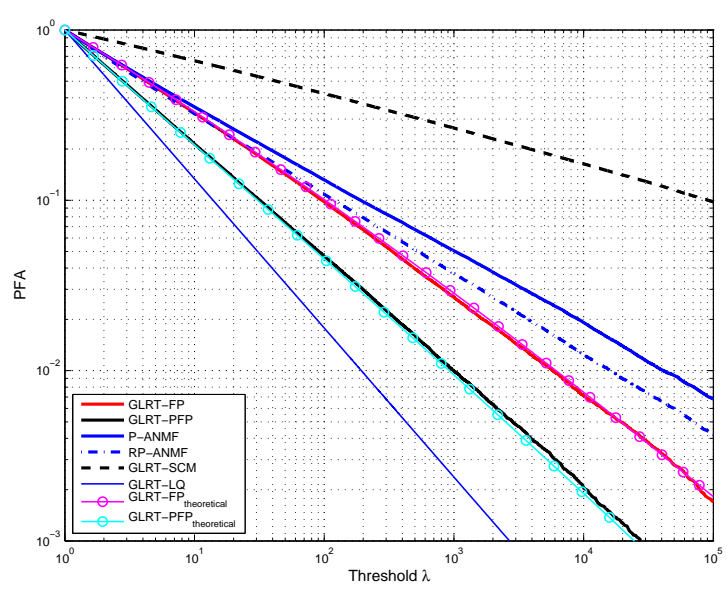

(a) Theoretical and experimental PFA-threshold curves for various non-Gaussian detectors.

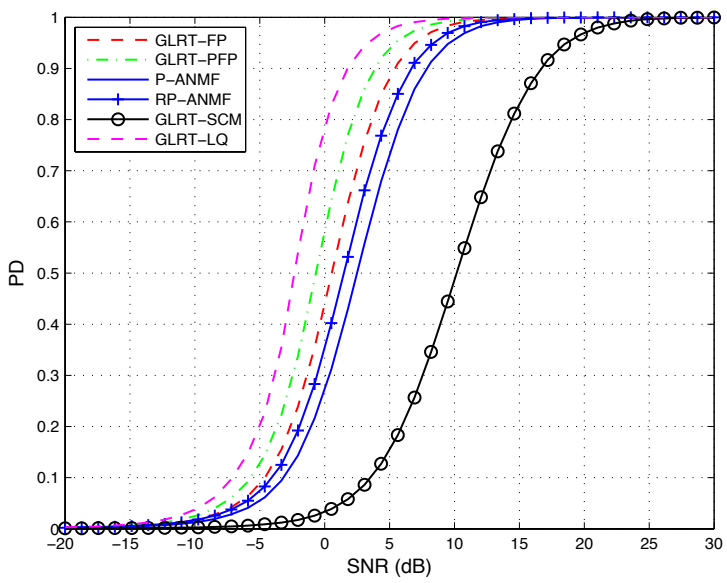

(b) Probability of Detection versus SNR for various non-Gaussian detectors

Fig. 5. Comparison between radar performance for various non-Gaussian detectors in simulated K-distributed clutter characterized by its parameter $\nu$ with $P_{f a}=10^{-3}, m=8, K=16$ and $\nu=0.2$. 


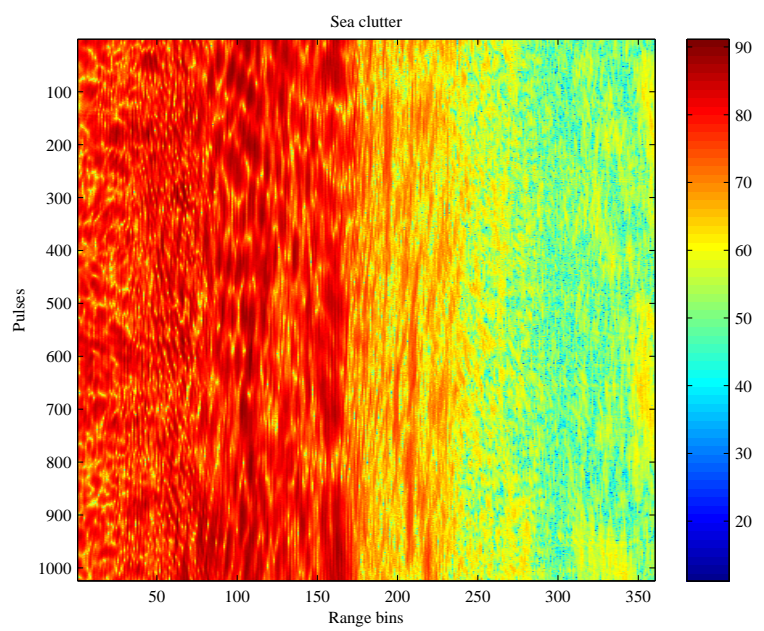

(a) Sea clutter data from an over the horizon radar.

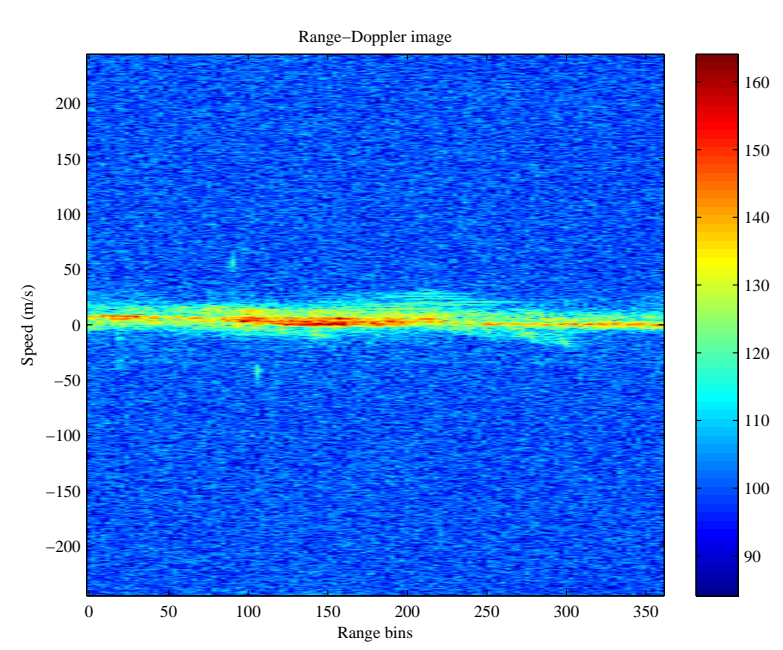

(b) Range-Doppler image of Atlantic Ocean sea clutter.

Fig. 6. Atlantic Ocean sea clutter data collected by the ONERA Over The Horizon Radar Nostradamus

Similar analyses performed on experimental sea-clutter data give the same conclusion. Figure 6(a) and figure 6(b) show the sea clutter signal (range bins versus Pulse Repetition Interval and Range-Doppler) collected by the operational Over The Horizon radar from the French Aerospace Lab (ONERA) illumining the Atlantic ocean, and its associated range-Doppler image. In this context, we use a set of $m=8$ pulses of the signal on the entire range bins group and $K=16$ reference range bins to estimate the CCM. Fig. 7(a) and Fig. 7(b) show the improvement in detection performance on these data and the agreement between theoretical (circle line) and practical (solid line) results.

\section{Conclusion}

In the radar detection framework, estimation of the CCM is a major procedure in the detection process. In many applications, since this matrix commonly exhibits a particular structure, we therefore introduced in this paper two adaptive detection tests which take into account the widespread persymmetric structure of the CCM. In both contexts of Gaussian and non-Gaussian environments, we have presented and analyzed new detectors based on a modified estimator of the CCM.

Under Gaussian assumption, the CCM estimator is developed based on the Maximum Likelihood procedure. The analytical distribution and some statistical properties of the corresponding detector, called the PS-AMF, have been established. These results are important since they enable a theoretical regulation 


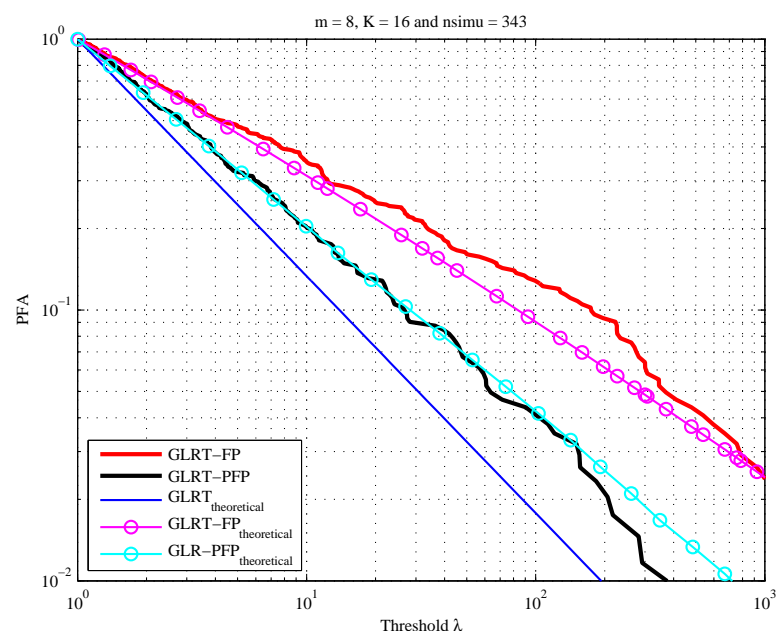

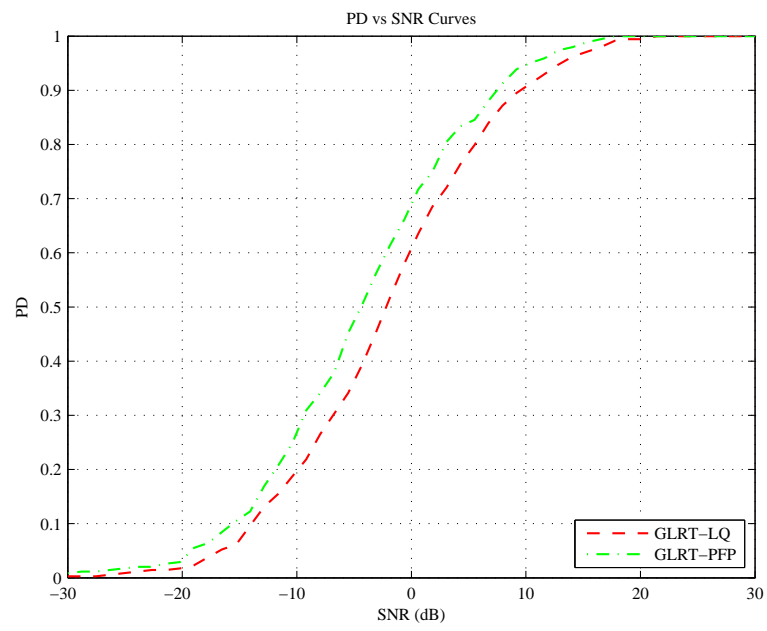

(a) Theoretical and experimental PFA-threshold curves for various non- (b) Probability of Detection versus SNR for the GLRT-FP and GLRTGaussian detectors PFP

Fig. 7. Comparison between radar performance for various non-Gaussian detectors in sea clutter data for $P_{f a}=10^{-2}, m=8$ and $K=16$.

of the false alarm, which is essential in the radar detection process.

The second detector is an extended version of the GLRT-LQ. It has been derived in the case of persymmetric non-Gaussian clutter modeled by SIRVs. After a transformation of the detection scheme, we have proposed an improved covariance matrix estimator, the PFP estimator. Its complete statistical analyses have exhibited good statistical performance. Moreover the corresponding GLRT-PFP detector has shown a wide improvement in terms of detection performance, as compared to the classical detectors.

Finally, all these theoretical results have been validated on simulations for both Gaussian and nonGaussian environments. Moreover, we have shown the validity and the good agreement between the theoretical and the experimental results, on both real ground and sea clutter data. Furthermore, the performance of the proposed detectors and the classical ones have been compared on these real data which highlight the improved performance of the former. These analysis have demonstrated the relevance and the advantage of exploiting the CCM structure. 


\section{ACKNOWLEDGMENT}

Authors would like to thank Thales Air System and DEMR/RBF unit of the "French Aerospace lab" for providing their experimental data.

\section{APPENDIX}

\section{A. Appendix A : Proof of the Proposition III.2}

We will use in this derivation the Bartlett matrix decomposition [28]. Let us set $\mathbf{e}_{1}=(1,0, \ldots, 0)^{\top}, \mathbf{R}=$ $\mathbf{R}^{\mathbf{1 / 2}} \mathbf{R}^{\top / \mathbf{2}}$ a factorization of $\mathbf{R}$ and $\mathbf{Q}$ a real unitary matrix such that $\mathbf{Q} \mathbf{R}^{-\mathbf{1} / \mathbf{2}} \mathbf{s}=\left(\mathbf{s}^{\top} \mathbf{R}^{-\mathbf{1}} s^{\mathbf{1} / \mathbf{2}} \mathbf{e}_{\mathbf{1}}\right.$. Note that the last equation is possible with $\mathbf{Q}$ real since $\mathbf{s}$ is itself real. Let us set

$$
\widehat{\mathbf{W}}=2 K \mathbf{Q} \mathbf{R}^{-\mathbf{1} / \mathbf{2}} \widehat{\mathbf{R}}_{\mathbf{P}} \mathbf{R}^{-\top / 2} \mathbf{Q}^{\top} \text { and } \mathbf{z}=\mathbf{Q R}^{-\mathbf{1} / \mathbf{2}} \mathbf{x}
$$

$\widehat{\mathbf{W}}$ is real Wishart distributed with $2 K$ degrees of freedom and parameter matrix $\mathbf{I}_{\mathbf{m}}, \mathbf{z} \sim \mathcal{C N}\left(\mathbf{0}, \mathbf{I}_{\mathbf{m}}\right)$. Then, the test statistic $\Lambda_{P S-A M F}(25)$ is equal to:

$$
\begin{aligned}
\Lambda_{P S-A M F} & =\frac{\left|\mathbf{s}^{\top} \widehat{\mathbf{R}}_{P}^{-1} \mathbf{x}\right|^{2}}{\mathbf{s}^{\top} \widehat{\mathbf{R}}_{P}^{-1} \mathbf{s}} \\
& =\frac{\left|\mathbf{s}^{\top} \mathbf{R}^{-\top / 2} \mathbf{Q}^{\top}\left(\mathbf{Q R}^{-1 / 2} \widehat{\mathbf{R}}_{P} \mathbf{R}^{-\top / 2} \mathbf{Q}^{\top}\right)^{-1} \mathbf{Q} \mathbf{R}^{-1 / 2} \mathbf{x}\right|^{2}}{\mathbf{s}^{\top} \mathbf{R}^{-\top / 2} \mathbf{Q}^{\top}\left(\mathbf{Q} \mathbf{R}^{-1 / 2} \widehat{\mathbf{R}}_{P} \mathbf{R}^{-\top / 2} \mathbf{Q}^{\top}\right)^{-1} \mathbf{Q} \mathbf{R}^{-1 / 2} \mathbf{s}} \\
& =2 K \frac{\left|\mathbf{e}_{1}^{\top} \widehat{\mathbf{W}}^{-1} \mathbf{z}\right|^{2}}{\mathbf{e}_{1}^{\top} \widehat{\mathbf{W}}^{-1} \mathbf{e}_{1}}
\end{aligned}
$$

which may be rewritten, for our statistical analysis, as:

$$
\Lambda_{P S-A M F}=2 K b a
$$

where:

$$
a=\frac{\left|\mathbf{e}_{1}^{\top} \widehat{\mathbf{W}}^{-1} \mathbf{x}\right|^{2}}{\mathbf{e}_{1}^{\top} \widehat{\mathbf{W}}^{-2} \mathbf{e}_{1}}, b=\left(\frac{\mathbf{e}_{1}^{\top} \widehat{\mathbf{W}}^{-2} \mathbf{e}_{1}}{\mathbf{e}_{1}^{\top} \widehat{\mathbf{W}}^{-1} \mathbf{e}_{1}}\right) .
$$

We will show that $a$ and $b$ are independent and we will derive their statistical distribution. Let us first investigate the distribution of $a$. By introducing the unitary vector $\mathbf{v}$, defined by:

$$
\mathbf{v}=\frac{1}{\left(\mathbf{e}_{1}^{\top} \widehat{\mathbf{W}}^{-2} \mathbf{e}_{1}\right)^{1 / 2}} \widehat{\mathbf{W}}^{-1} \mathbf{e}_{1},
$$

$a$ may be rewritten as:

$$
a=\left|\mathbf{v}^{\top} \mathbf{x}\right|^{2}
$$


It follows that the conditional distribution of $2 a$ given $\widehat{\mathbf{W}}$ is a Chi square distribution with 2 degrees of freedom denoted by $\chi_{2}^{2}$. This distribution does not involve $\widehat{\mathbf{W}}$, and

$$
a \sim \frac{1}{2} \chi_{2}^{2}
$$

is therefore independent of $\widehat{\mathbf{W}}$ and consequently of $b$.

Now, to derive the PDF of $b$, we use the Bartlett matrix decomposition $\widehat{\mathbf{W}}=\mathbf{U} \cdot \mathbf{U}^{\top}$ where $\mathbf{U}=$ $\left(u_{i j}\right)_{1 \leq i \leq j \leq m}$ is an upper triangular matrix whose random elements are independent and distributed as:

$$
u_{i, i}^{2} \sim \chi_{2 K+i-m}^{2} \text { and } u_{i, j} \sim \mathcal{N}(0,1) \text { for } i<j .
$$

Let $u_{i, j}^{\prime}$ be the elements of the matrix $\mathbf{U}^{-1}$ which is itself upper-triangular. By noticing that $\mathbf{U}^{-1} \mathbf{e}_{1}=$ $u_{1,1}^{\prime} \mathbf{e}_{1}$, we have :

$$
\begin{aligned}
b & =\frac{\mathbf{e}_{1}^{\top} \widehat{\mathbf{W}}^{-2} \mathbf{e}_{1}}{\mathbf{e}_{1}^{\top} \widehat{\mathbf{W}}^{-1} \mathbf{e}_{1}} \\
& =\frac{\mathbf{e}_{1}^{\top} \mathbf{U}^{-\top} \mathbf{U}^{-1} \mathbf{U}^{-\top} \mathbf{U}^{-1} \mathbf{e}_{1}}{\mathbf{e}_{1}^{\top} \mathbf{U}^{-\top} \mathbf{U}^{-1} \mathbf{e}_{1}} \\
& =\left\|\mathbf{e}^{\top} \mathbf{U}^{-1}\right\|^{2} \\
& =\sum_{j=1}^{m} u_{1, j}^{\prime 2}
\end{aligned}
$$

We are thus lead to investigate the distribution of the squared norm of the first row of $\mathbf{U}^{-1}$. Let $\mathbf{u}_{1, k}^{\prime}$ be the $k$-th order vector whose components are the $k$ first elements of the first row of $\mathbf{U}^{-1}$ and $\mathbf{u}_{i, j}$ the $i$-th order vector the components of which are the $i$ first elements of the $j$-th column of $\mathbf{U}$. The definition of $\mathbf{U}^{-1}$, i.e. $\mathbf{U}^{-1} \mathbf{U}=\mathbf{I}_{m}$, allows to determine recursively its elements according to:

$$
u_{1,1}^{\prime}=u_{1,1}^{-1}
$$

and

$$
u_{1, k+1}^{\prime}=\frac{-\mathbf{u}_{1, k}^{\prime \top} \mathbf{u}_{k, k+1}}{u_{k+1, k+1}} \text { for } 1 \leqslant k<m .
$$

It follows that $\mathbf{u}_{1, k}^{\prime}$ is independent of the $u_{i, j}$ 's for $j>k$. Now we have from the above equation:

$$
\begin{aligned}
u_{1, k+1}^{\prime 2} & =\frac{\left|\mathbf{u}_{1, k}^{\prime \top} \mathbf{u}_{k, k+1}\right|^{2}}{u_{k+1, k+1}^{2}} \\
& =\frac{\left|\mathbf{u}_{1, k}^{\prime \top} \mathbf{u}_{k, k+1}\right|^{2}}{\left\|\mathbf{u}_{1, k}^{\prime}\right\|^{2}} \frac{\left\|\mathbf{u}_{1, k}^{\prime}\right\|^{2}}{u_{k+1, k+1}^{2}} \\
& ==\alpha_{k} \frac{\left\|\mathbf{u}_{1, k}^{\prime}\right\|^{2}}{u_{k+1, k+1}^{2}} .
\end{aligned}
$$


The conditional distribution of $\alpha_{k}$ given $\mathbf{u}_{1, k}^{\prime}$ is a Chi square distribution with 1 degree of freedom denoted by $\chi_{1}^{2}$. This distribution does not involve $\mathbf{u}_{1, k}^{\prime}$ and $\alpha_{k} \sim \chi_{1}^{2}$ is therefore independent of $\mathbf{u}_{1, k}^{\prime}$. Moreover, $\alpha_{k}$ is independent of $u_{i, j}$ for $i$ and $j>k$. Now, notice from Eqn. (48) that $b=\left\|\mathbf{u}_{1, m}^{\prime}\right\|^{2}$. Since $\left\|\mathbf{u}_{1, m}^{\prime}\right\|^{2}=\left\|\mathbf{u}_{1, m-1}^{\prime}\right\|^{2}+u_{1, m}^{\prime 2}$, one has therefore:

$$
b=\left\|\mathbf{u}_{m-1}^{\prime}\right\|^{2}\left(1+\frac{\alpha_{m-1}}{u_{m, m}^{2}}\right)=\frac{1}{u_{1,1}^{2}} \prod_{k=2}^{m}\left(1+\frac{\alpha_{k-1}}{u_{k, k}^{2}}\right),
$$

where the $\alpha_{k}$ 's are independent, independent of the $u_{k, k}^{2}$ 's and $\chi_{1}^{2}$-distributed. Since $\left(1+\frac{\alpha_{k-1}}{u_{k, k}^{2}}\right)^{-1} \sim$ $\beta_{1}\left(\frac{2 K-m+k}{2}, \frac{1}{2}\right)$, we have from [29]:

$$
b=\frac{1}{u_{1,1}^{2}} \frac{1}{\prod_{k=2}^{m} \beta_{1}\left(\frac{2 K-m+k}{2}, \frac{1}{2}\right)} \sim \frac{1}{\chi_{2 K-m+1}^{2}} \frac{1}{\beta_{1}\left(\frac{2 K-m+2}{2}, \frac{m-1}{2}\right)},
$$

where the $\beta_{1}$ 's are independent (first kind) Beta distributed random variables. Finally we obtain from Eqn. (45), Eqn. (46) and Eqn. (49):

$$
\Lambda_{P S-A M F} \sim K \frac{\chi_{2}^{2}}{\chi_{2 K-m+1}^{2}} \frac{1}{\beta_{1}\left(\frac{2 K-m+2}{2}, \frac{m-1}{2}\right)}
$$

which can be rewritten in terms of an F-distributed random variable:

$$
\Lambda_{P S-A M F} \sim K \frac{2}{2 K-m+1} F(2,2 K-m+1) \frac{1}{\beta_{1}\left(\frac{2 K-m+2}{2}, \frac{m-1}{2}\right)}
$$

Let us now derive the PFA-threshold relation. From [13], page 946:

$$
\begin{aligned}
P F A & =\operatorname{Pr}\left(\Lambda_{P S-A M F}>\lambda\right) \\
& =\operatorname{Pr}\left(F(2,2 K-m+1)>\frac{2 K-m+1}{2} \frac{\lambda}{K} \beta_{1}\left(\frac{2 K-m+2}{2}, \frac{m-1}{2}\right)\right) \\
& =\int_{0}^{1}\left(\frac{1}{1+\frac{\lambda}{K} x}\right)^{\frac{2 K-m+1}{2}} f_{\nu 1, \nu 2}(x) d x
\end{aligned}
$$

where $f_{\nu 1, \nu 2}$ is the PDF of a first kind beta random variable with parameters $\nu 1=\frac{2 K-m+2}{2}$ and $\nu 2=$ $\frac{m-1}{2}$. We finally obtain:

$$
P F A=\frac{1}{B\left(\frac{2 K-m+2}{2}, \frac{m-1}{2}\right)} \int_{0}^{1}\left(\frac{1}{1+\frac{\lambda}{K} . x}\right)^{\frac{2 K-m+1}{2}} x^{\frac{2 K-m+2}{2}-1}(1-x)^{\frac{m-1}{2}-1} d x
$$


which may be expressed in terms of the hypergeometric function [13] page 558:

$$
P_{f a}={ }_{2} F_{1}\left(\frac{2 K-m+1}{2}, \frac{2 K-m+2}{2} ; \frac{2 K+1}{2} ;-\frac{\lambda}{K}\right) .
$$

The negative derivative of $P_{f a}$ with respect to $\lambda$ yields the PDF of $\Lambda_{P S-A M F}$. By using the expression of the derivative of hypergeometric functions given in [13] page 557, we obtain Eqn. (27) which concludes the proof.

B. Appendix B : Proof of the proposition III.5

From theorem III.4, $\Lambda_{G L R T-P F P}$ has the same asymptotic distribution as

$$
\begin{aligned}
\Lambda & =\frac{\left|\mathbf{e}_{1}^{\top} \widehat{\mathbf{W}}^{-1} \mathbf{w}\right|^{2}}{\left(\mathbf{e}_{1}^{\top} \widehat{\mathbf{W}}^{-1} \mathbf{e}_{1}\right)\left(\mathbf{w}^{H} \widehat{\mathbf{W}}^{-1} \mathbf{w}\right)} \\
& =\frac{\left|\mathbf{e}_{1}^{\top} \widehat{\mathbf{W}}^{-1}(\sqrt{2} \mathbf{w})\right|^{2}}{\left(\mathbf{e}_{1}^{\top} \widehat{\mathbf{W}}^{-1} \mathbf{e}_{1}\right)\left(\sqrt{2} \mathbf{w}^{H} \widehat{\mathbf{W}}^{-1} \sqrt{2} \mathbf{w}\right)},
\end{aligned}
$$

where $(\sqrt{2} \mathbf{w})=\mathbf{w}_{1}+i \mathbf{w}_{2}$ with $\mathbf{w}_{1}$ and $\mathbf{w}_{2}$ uncorrelated and $\mathcal{N}(\mathbf{0}, \mathbf{I})$ distributed.

Thus

$$
\Lambda=\frac{\left|\mathbf{e}_{1}^{\top} \widehat{\mathbf{W}}^{-1} \mathbf{w}_{1}\right|^{2}+\left|\mathbf{e}_{1}^{\top} \widehat{\mathbf{W}}^{-1} \mathbf{w}_{2}\right|^{2}}{\left(\mathbf{e}_{1}^{\top} \widehat{\mathbf{W}}^{-1} \mathbf{e}_{1}\right)\left(\mathbf{w}_{1}^{\top} \widehat{\mathbf{W}}^{-1} \mathbf{w}_{1}+\mathbf{w}_{2}^{\top} \widehat{\mathbf{W}}^{-1} \mathbf{w}_{2}\right)}
$$

For large $K, \widehat{\mathbf{W}}$ is real Wishart distributed with $K^{\prime}=\frac{m}{m+1} 2 K$ degrees of freedom. The vectors $\mathbf{w}_{1}$ and $\mathbf{w}_{2}$ can be decomposed on an orthonormal vectors triplet $\left(\mathbf{e}_{1}, \mathbf{f}_{2}, \mathbf{f}_{3}\right)$ :

$$
\begin{aligned}
& \mathbf{w}_{1}=\alpha_{1} \mathbf{e}_{1}+\alpha_{2} \mathbf{f}_{2} \\
& \mathbf{w}_{2}=\beta_{1} \mathbf{e}_{1}+\beta_{2} \mathbf{f}_{2}+\beta_{3} \mathbf{f}_{3} .
\end{aligned}
$$

where $\alpha_{1}, \beta_{1}$ and $\beta_{2}$ are $\mathcal{N}(0,1)$ distributed, $\alpha_{2}^{2}$ is $\chi_{m-1}^{2}$ distributed and $\beta_{3}^{2}$ is $\chi_{m-2}^{2}$ distributed. Moreover $\alpha_{1}, \alpha_{2}, \beta_{1}, \beta_{2}, \beta_{3}$ are independent and independent of $\left(\mathbf{f}_{2}, \mathbf{f}_{3}\right)$.

Let $\left(\mathbf{e}_{1}, \mathbf{e}_{2}, \ldots, \mathbf{e}_{m}\right)$ be the canonical basis. Using an appropriate rotation $\mathbf{G}$ such that $\mathbf{G}\left(\mathbf{e}_{1}, \mathbf{f}_{2}, \mathbf{f}_{3}\right)=$ $\left(\mathbf{e}_{1}, \mathbf{e}_{2}, \mathbf{e}_{3}\right)$, we have

$$
\begin{aligned}
\mathbf{G w}_{1} & =\alpha_{1} \mathbf{e}_{1}+\alpha_{2} \mathbf{e}_{2} \\
& =\mathbf{v}_{1}
\end{aligned}
$$




$$
\begin{aligned}
\mathbf{G w}_{2} & =\beta_{1} \mathbf{e}_{1}+\beta_{2} \mathbf{e}_{2}+\beta_{3} \mathbf{e}_{3} \\
& =\mathbf{v}_{2}
\end{aligned}
$$

and $\Lambda$ can be rewritten as

$$
\Lambda=\frac{\left|\mathbf{e}_{1}^{\top} \widehat{\mathbf{Z}}^{-1} \mathbf{v}_{1}\right|^{2}+\left|\mathbf{e}_{1}^{\top} \widehat{\mathbf{Z}}^{-1} \mathbf{v}_{2}\right|^{2}}{\left(\mathbf{e}_{1}^{\top} \widehat{\mathbf{Z}}^{-1} \mathbf{e}_{1}\right)\left(\mathbf{v}_{1}^{\top} \widehat{\mathbf{Z}}^{-1} \mathbf{v}_{1}+\mathbf{v}_{2}^{\top} \widehat{\mathbf{Z}}^{-1} \mathbf{v}_{2}\right)}
$$

where $\widehat{\mathbf{Z}}=\mathbf{G} \widehat{\mathbf{W}} \mathbf{G}^{\top}$.

Conditionally (and unconditionally) to $\mathbf{G}, \widehat{\mathbf{Z}}$ is Wishart distributed with $K^{\prime}$ degrees of freedom and parameter matrix I. Let $\widehat{\mathbf{Z}}=\mathbf{L}^{\top} \mathbf{L}$ be the Bartlett's decomposition of $\widehat{\mathbf{Z}}$ [28] where $\mathbf{L}=\left(l_{i, j}\right)_{1 \leq i \leq j \leq m}$ is a lower triangular matrix whose non-zeros random elements are independent and distributed as:

$$
l_{i, i}^{2} \sim \chi_{K^{\prime}+i-m}^{2} \text { and } l_{i, j} \sim \mathcal{N}(0,1) \text { for } i>j .
$$

Let $l_{i, j}^{\prime}$ be the elements of the matrix $\mathbf{L}^{-1}$ which is lower-triangular itself. The following elements of $\mathbf{L}^{-1}$ are involved in Eqn. (59):

$$
l_{11}^{\prime}=\frac{1}{l_{11}}, l_{22}^{\prime}=\frac{1}{l_{22}}, l_{21}^{\prime}=-\frac{l_{21}}{l_{11} l_{22}}, l_{33}^{\prime}=\frac{1}{l_{33}}, l_{32}^{\prime}=-\frac{l_{32}}{l_{22} l_{33}}, l_{31}^{\prime}=-\frac{1}{l_{11}}\left(\frac{l_{31}}{l_{33}}-\frac{l_{32} l_{21}}{l_{22} l_{33}}\right) .
$$

From Eqn. (59), we define:

$$
\alpha=\frac{\left|\mathbf{e}_{1}^{\top} \widehat{\mathbf{Z}}^{-1} \mathbf{v}_{1}\right|^{2}+\left|\mathbf{e}_{1}^{\top} \widehat{\mathbf{Z}}^{-1} \mathbf{v}_{2}\right|^{2}}{\mathbf{e}_{1}^{\top} \widehat{\mathbf{Z}}^{-1} \mathbf{e}_{1}}
$$

which can be rewritten as:

$$
\begin{aligned}
\alpha & =\frac{\left|\mathbf{e}_{1}^{\top} \mathbf{L}^{-1} \mathbf{L}^{-\top} \mathbf{G} \mathbf{w}_{1}\right|^{2}+\left|\mathbf{e}_{1}^{\top} \mathbf{L}^{-1} \mathbf{L}^{-\top} \mathbf{G} \mathbf{w}_{2}\right|^{2}}{\mathbf{e}_{1}^{\top} \mathbf{L}^{-1} \mathbf{L}^{-\top} \mathbf{e}_{1}}, \\
& =\left(\alpha_{1} l_{11}^{\prime}+\alpha_{2} l_{21}^{\prime}\right)^{2}+\left(\beta_{1} l_{11}^{\prime}+\beta_{2} l_{21}^{\prime}+\beta_{3} l_{31}^{\prime}\right)^{2},
\end{aligned}
$$

and

$$
\beta=\mathbf{v}_{1}^{\top} \widehat{\mathbf{Z}}^{-1} \mathbf{v}_{1}+\mathbf{v}_{2}^{\top} \widehat{\mathbf{Z}}^{-1} \mathbf{v}_{2}
$$

which can be rewritten as:

$$
\begin{aligned}
\beta & =\mathbf{v}_{1}^{\top} \mathbf{L}^{-1} \mathbf{L}^{-\top} \mathbf{v}_{1}+\mathbf{v}_{2}^{\top} \mathbf{L}^{-1} \mathbf{L}^{-\top} \mathbf{v}_{2} \\
& =\alpha+\left(l_{22}^{\prime} \alpha_{2}\right)^{2}+\left(l_{22}^{\prime} \beta_{2}+l_{32}^{\prime} \beta_{3}\right)^{2}+\left(l_{33}^{\prime} \beta_{3}\right)^{2}
\end{aligned}
$$


We deduce that $\Lambda=\frac{\alpha}{\beta}=\frac{F}{1+F}$ with

$$
\begin{aligned}
F & =\frac{\left(\alpha_{1} l_{11}^{\prime}+\alpha_{2} l_{21}^{\prime}\right)^{2}+\left(\beta_{1} l_{11}^{\prime}+\beta_{2} l_{21}^{\prime}+\beta_{3} l_{31}^{\prime}\right)^{2}}{\left(l_{22}^{\prime} \alpha_{2}\right)^{2}+\left(l_{22}^{\prime} \beta_{2}+l_{32}^{\prime} \beta_{3}\right)^{2}+\left(l_{33}^{\prime} \beta_{3}\right)^{2}} \\
& =\frac{\left(\alpha_{1} l_{22}-\alpha_{2} l_{21}\right)^{2}+\left(\beta_{1} l_{22}-\beta_{2} l_{21}-\beta_{3} \frac{l_{31}}{l_{33}} l_{22}+\frac{l_{32} l_{21}}{l_{33}} \beta_{3}\right)^{2}}{\left(l_{11} \alpha_{2}\right)^{2}+\left(\frac{l_{11} l_{22}}{l_{33}} \beta_{3}\right)^{2}+l_{11}^{2}\left(\beta_{2}-\frac{\beta_{3}}{l_{33}} l_{32}\right)^{2}} .
\end{aligned}
$$

In this equation, we have

$$
\begin{aligned}
\left(\beta_{1} l_{22}-\beta_{2} l_{21}-\beta_{3} \frac{l_{31}}{l_{33}} l_{32}+\frac{l_{32} l_{21}}{l_{33}} \beta_{3}\right)^{2} & \left.=\left(l_{22}\left(\beta_{1}-\frac{\beta_{3}}{l_{33}} l_{31}\right)-l_{21}\left(\beta_{2}-\frac{\beta_{3}}{l_{33}} l_{32}\right)\right)\right)^{2} \\
& =\left(1+\left(\frac{\beta_{3}}{l_{33}}\right)^{2}\right)\left(l_{22} a-l_{21} b\right)^{2}
\end{aligned}
$$

with $a=\frac{1}{\sqrt{1+\left(\frac{\beta_{3}}{l_{33}}\right)^{2}}}\left(\beta_{1}-\frac{\beta_{3}}{l_{33}} l_{31}\right)$ and $b=\frac{1}{\sqrt{1+\left(\frac{\beta_{3}}{l_{33}}\right)^{2}}}\left(\beta_{2}-\frac{\beta_{3}}{l_{33}} l_{32}\right)$. Conditionally to $\beta_{3}$ and $l_{33}, a$ and $b$ are independent and $\mathcal{N}(0,1)$ distributed. Since their distribution does not involve $\beta_{3}$ and $l_{33}, a$ and $b$ are also independent of $\beta_{3}$ and $u_{33}$.

By replacing it in Eqn. (61), we finally obtain

$$
F=\frac{\left(\alpha_{1} l_{22}-\alpha_{2} l_{21}\right)^{2}+\left(1+\left(\frac{\beta_{3}}{l_{33}}\right)^{2}\right)\left(a l_{22}-b l_{21}\right)^{2}}{\left(\alpha_{2} l_{11}\right)^{2}+\left(l_{11} l_{22} \frac{\beta_{3}}{l_{33}}\right)^{2}+l_{11}^{2}\left(1+\left(\frac{\beta_{3}}{l_{33}}\right)^{2}\right) b^{2}}
$$

where all the following random variables are independent and distributed according to:

$$
a, b, \alpha_{1}, l_{21} \sim \mathcal{N}(0,1), \alpha_{2}^{2} \sim \chi_{m-1}^{2}, \beta_{3}^{2} \sim \chi_{m-2}^{2}, l_{11}^{2} \sim \chi_{K^{\prime}-m+1}^{2}, l_{22}^{2} \sim \chi_{K^{\prime}-m+2}^{2}, l_{33}^{2} \sim \chi_{K^{\prime}-m+3}^{2},
$$

with $K^{\prime}=\frac{m}{m+1} 2 K$ which concludes the proof.

\section{REFERENCES}

[1] J.P. Burg, D.G. Luenberger, and D.L. Wenger, "Estimation of Structured Covariance Matrices," Proc. of the IEEE, vol. 70, pp. 963-974, September 1982.

[2] D.R. Fuhrmann, "Application of Toeplitz Covariance Estimation to Adaptive Beamforming and Detection," IEEE Trans. on SP, vol. 39, pp. 2194-2198, October 1991.

[3] R. Nitzberg and J.R. Burke, "Application of Maximum Likelihood Estimation of Persymmetric Covariance Matrices to Adaptive Detection,” IEEE Trans. on AES, vol. 25, pp. 124-127, January 1980.

[4] L. Cai and H. Wang, "A Persymmetric Multiband GLR Algorithm,” IEEE Trans. on AES, pp. 806-816, July 1992. 
[5] E. Conte and A. De Maio, "Exploiting Persymmetry for CFAR Detection in Compound-Gaussian Clutter," IEEE Trans. on AES, vol. 39, pp. 719-724, April 2003.

[6] E. Conte and A. De Maio, "Mitigation Techniques for Non-Gaussian Sea Clutter," IEEE Journal of Oceanic Engineering, vol. 29, pp. 284-302, December 2003.

[7] F. Gini and M. V Greco, "Covariance matrix estimation for CFAR detection in correlated heavy tailed clutter," Signal Processing, special section on Signal Processing with Heavy Tailed Distributions, vol. 82, pp. 1847-1859, December 2002.

[8] E. Conte, A. De Maio, and G. Ricci, "Recursive estimation of the covariance matrix of a compound-Gaussian process and its application to adaptive CFAR detection," IEEE Trans. on SP, vol. 50, pp. 1908-1915, August 2002.

[9] G. Pailloux, P. Forster, J.P. Ovarlez, and F. Pascal, “On Persymmetric Covariance Matrices in Adaptive Detection,” IEEE ICASSP 2008, pp. 2305-2308, April 2008.

[10] G. Pailloux, J.P. Ovarlez, F. Pascal, and P. Forster, "A SIRV-CFAR Adaptive Detector Exploiting Persymmetric Clutter Covariance Structure,” IEEE RadarConf 2008, pp. 1139-1144, May 2008.

[11] F.C. Robey, D.R. Fuhrmann, E.J. Kelly, and R. Nitzberg, “A CFAR Adaptive Matched Filter Detector," IEEE Trans. on AES, vol. 23, pp. 208-216, January 1992.

[12] S. Kraut, L.L. Scharf, and L.T. Mc Whorter, “Adaptive Subspace Detector,” IEEE Trans. on SP, vol. 49, pp. 1-16, January 2001.

[13] M. Abramovitch and I.A. Stegun, "Handbook of Mathematical Functions," National Bureau of standard, vol. AMS 55, June 1964.

[14] J.B. Billingsley, “Ground Clutter Measurement for Surface Sited Radar,” MIT Technical report 780, February 1993.

[15] K. Yao, “A Representation Theorem and its Applications to Spherically Invariant Random Processes," IEEE Trans. on IT, vol. 19, pp. 600-608, September 1973.

[16] M. Rangaswamy, D.D. Weiner, and A. Ozturk, "Non-Gaussian vector identification using spherically invariant random processes,” IEEE Trans. on AES, vol. 29, pp. 111-124, January 1993.

[17] F. Gini, "Sub-Optimum Coherent Radar Detection in a Mixture of K-Distributed and Gaussian Clutter," IEE Proc. on Radar, Sonar Navig., vol. 144, pp. 39-48, February 1997.

[18] E. Conte, M. Lops, and G. Ricci, “Asymptotically Optimum Radar Detection in Compound-Gaussian Clutter," IEEE Trans. on AES, vol. 31, pp. 617-625, April 1995.

[19] L.L. Scharf and L.T. McWhorter, “Adaptive Matched Subspace Detectors and Adaptive Coherence Estimators,” Proceedings of the 30th Asilomar Conference on Signals, Systems, and Computers, vol. 2, pp. 1114-1117, November 1996.

[20] F. Pascal, Y. Chitour, J.P. Ovarlez, P. Forster, and P. Larzabal, "Covariance Structure Maximum Likelihood Estimates in Compound Gaussian Noise: Existence and Algorithm Analysis," IEEE Trans. on SP, vol. 56, pp. 34-38, January 2008.

[21] F. Pascal, P. Forster, J.P. Ovarlez, and P. Larzabal, "Performance Analysis of Covariance Matrix Estimate in Impulsive Noise," IEEE Trans. on SP, pp. 2206-2217, June 2008.

[22] F Pascal, J-P Ovarlez, P Forster, and P Larzabal, "Constant false alarm rate detection in spherically invariant random processes," in Proc. of the European Signal Processing Conf., EUSIPCO-04, Vienna, September 2004, pp. $2143-2146$.

[23] H L Van Trees, Detection, Estimation and Modulation Theory, Part IV: Optimum Array Processing, John Wiley \& Sons, New York, 2002.

[24] I. Reed, Y.S.Gau, and T.K. Truong, "CFAR Detection and Estimation for STAP Radar," IEEE Trans. on AES, vol. 34, pp. 722-735, July 1998. 
[25] F. Pascal, J.P. Ovarlez, P. Forster, and P. Larzabal, "On a SIRV-CFAR Detector with Radar Experimentations in Impulsive Noise," Proc. EUSIPCO, September 2006.

[26] D.J. Sheskin, "Parametric and non-parametric statistical procedures," Chapmann and Hall/CRC books, New York, 2004.

[27] R.S. Raghavan, “A Method for Estimating Parameters of K-Distributed Clutter,” IEEE Trans. on AES, vol. 27, pp. 238-246, March 1991.

[28] R.J. Muirhead, “Aspects of Multivariate Statistical Theory,” Wiley, New-York, 1982.

[29] M.V. Jambunathan, "Some Properties of Beta and Gamma Distributions," The annals of mathematical statistics, vol. 25, pp. 401-405, June 1954. 